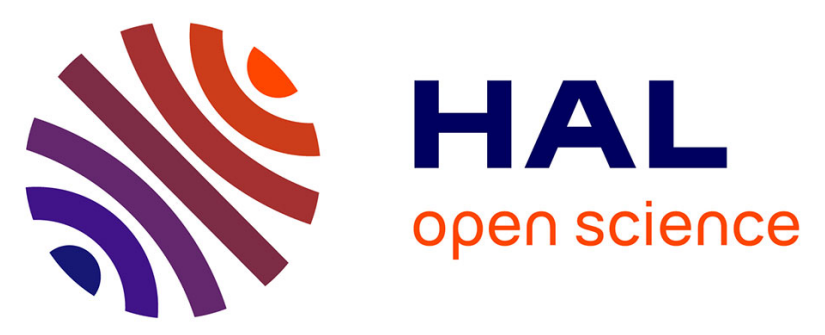

\title{
Large eddy simulation of a turbulent swirling premixed flame coupling the TFLES model with a dynamic wrinkling formulation
}

\author{
Pedro Volpiani, Schmitt Thomas, Denis Veynante
}

\section{To cite this version:}

Pedro Volpiani, Schmitt Thomas, Denis Veynante. Large eddy simulation of a turbulent swirling premixed flame coupling the TFLES model with a dynamic wrinkling formulation. Combustion and Flame, 2017, 180, pp.124 - 135. 10.1016/j.combustflame.2017.02.028 . hal-01643053

\section{HAL Id: hal-01643053 https://hal.science/hal-01643053}

Submitted on 22 Nov 2021

HAL is a multi-disciplinary open access archive for the deposit and dissemination of scientific research documents, whether they are published or not. The documents may come from teaching and research institutions in France or abroad, or from public or private research centers.
L'archive ouverte pluridisciplinaire HAL, est destinée au dépôt et à la diffusion de documents scientifiques de niveau recherche, publiés ou non, émanant des établissements d'enseignement et de recherche français ou étrangers, des laboratoires publics ou privés. 


\title{
Large eddy simulation of a turbulent swirling premixed flame coupling the TFLES model with a dynamic wrinkling formulation
}

\author{
P. S. Volpiani, T. Schmitt, D. Veynante \\ Laboratoire EM2C, CNRS/Ecole CentraleSupélec, Université Paris-Saclay, Grande Voie \\ des Vignes 92295 Chatenay-Malabry, France
}

\begin{abstract}
Dynamic models that take advantage of the known resolved scales to automatically adjust the model parameters have proved to be very effective in large eddy simulations (LES). Global (uniform parameter evolving only with time) and local (parameter evolving both in space and time) dynamic formulations for the flame wrinkling factor are combined with the Thickened Flame (TFLES) model and simulations of the semi-industrial PRECCINSTA burner studied experimentally by Meier et al. [1] are performed for the stable and unstable configurations. The global formulation predicts a time-dependent model exponent that remains close to 0.5 for the stable flame and oscillates strongly around 0.8 for the pulsating flame. The local formulation adapts the model parameter locally and automatically damps the wrinkling factor in the vicinity of walls, contrary to the global formulation requiring a wall law. The usual non-dynamic approach with an appropriate parameter is found to capture flow statistics of the stable flame with good accuracy, both in terms of Favre and quasi-Reynolds averages. However, the self-excited mode of the pulsating flame is predicted only with the dynamic formalism. The fractal
\end{abstract}


dimension of the unstable flame is found to vary locally and depends on the phase within the period of oscillation. Dynamic models may then play an important role in the prediction of combustion instabilities.

Keywords: Turbulent combustion, Large eddy simulation, Dynamic

modeling, Combustion instabilities, PRECCINSTA burner

\section{Introduction}

Dynamic models have proved to be a powerful tool in Large Eddy Simulations (LES). The basic idea of such models, developed to describe sub-grid scale momentum transport [2], is to take advantage of the known instantaneous resolved large scales to automatically adjust model parameters. The instantaneous resolved field is filtered at a test filter scale larger than the original LES filter. The model is then assumed to hold at both scales and model parameters are solutions of a Germano-like equation.

While dynamic models are now routinely used for momentum transport, their application to reaction rate modeling in combusting flows remains rather scarce and often restricted to simple flow configurations (flame embedded in a homogeneous isotropic turbulent flow [3-5], jet flames [6-9]). Few studies treat relatively more complex geometries and realistic burners [1013]. One main reason explains this situation. Combustion and turbulence behave very differently: most of the turbulence energy is resolved in LES, a way to check simulation quality [14], while combustion is mainly a sub-grid scale phenomenon, possibly leading to an ill-posed problem when looking for a linear parameter in a dynamic procedure [4].

Flame front wrinkling factors, quantifying flame / turbulence interactions 
in terms of ratio of total to resolved flame surfaces in the filter volume enter directly flame surface density (FSD) [15], thickened flame (TFLES) [16, 17] and F-TACLES [18] models. They also may be used to model the sub-grid scale turbulence flame speed in level-set formalism [6, 19]. Charlette et al. [4] proposed a global dynamic formulation where the spatially-uniform timedependent exponent parameter of a fractal wrinkling factor expression is determined automatically. Wang et al. [5, 7] have shown the ability of such a dynamic model to reproduce a statistically steady jet flame [20] and the transient ignition of a flame kernel [21] under several operating conditions. The TFLES model was used in the first case while the second retained the Boger et al. algebraic FSD model [15]. Knikker et al. [22, 23] proposed a Dynamic Flame Surface Density (DFSD) model based on a fractal analysis $[24,25]$ and on a similarity assumption [26]. This model was validated a priori from experimental data and, more recently, tested a posteriori by Ibrahim et al. [12] and Gubba et al. [13] to simulate the propagation of a turbulent premixed flame through obstacles in a laboratory scale combustion chamber.

Using the level-set formalism, Knudsen and Pitsch [6] performed simulations where the parameter of a model expression for the sub-grid scale turbulent flame speed depends on both space coordinates and time. Schmitt et al. $[8,10]$ adopted a similar strategy coupling a dynamic fractal wrinkling factor expression with the tabulated chemistry F-TACLES method. They simulated the Tecflam turbulent swirl burner $[27,28]$ and, later, turbulent Bunsen flames [20] over three different operating conditions. Volpiani et al. [9] simulated the F3 jet flame studied by Chen et al. [20] and investigated the 
influence of physical and numerical characteristics of a flame wrinkling factor dynamic model for both, global (i.e. spatially-uniform time-dependent) and local (space and time dependent), model parameters. A similar local flame wrinkling factor dynamic formalism, combined with the Boger et al. model [15], gives very promising results to predict the development of a flame kernel in an internal combustion engine [29], even if an adapted ignition model remains to be developed.

Other authors applied the dynamic formalism to compute variances and scalar dissipation rates of a mixture fraction, that enter non-premixed combustion models [30-34]. These procedures can be denoted "indirect approaches", to differ from the previous one that involve directly reaction rate terms.

The goal of this paper is to apply the TFLES combustion model coupled with global and local dynamic procedures in simulations of a realistic burner configuration and to assess the influence of the dynamic model in the prediction of combustion instabilities. The chosen configuration is the PRECCINSTA swirl burner derived from a gas turbine designed by Turbomeca. This configuration has been the subject of many experimental $[1,35]$ and numerical [36-41] studies. Experimental studies using the PRECCINSTA burner evidenced two combustion regimes [1]: a quiet flame at equivalence ratio $\phi=0.83$ and a pulsating flame at $\phi=0.70$. Numerical simulations commonly assume perfect mixing between fuel and air at the combustion chamber inlet because in the experiment methane is injected in the swirler, far upstream of the combustor. However, in the configuration where self-excited combustion oscillations are found, simulations assuming perfect mixing fail 
to predict the combustion instability [42].

The paper is organized as follows: basic concepts of the TFLES combustion model are first reviewed. The dynamic procedure is then briefly discussed. Experimental and numerical set-ups are presented. Numerical results are then compared to experiments and discussed for both stable and unstable cases. Conclusions are drawn.

\section{Modeling}

\subsection{The thickened flame model (TFLES)}

Flames are artificially thickened to be resolved on the numerical grid by multiplying diffusion and dividing reaction rates by a thickening factor $\mathcal{F}$ but conserving the laminar flame speed $S_{L}[43,44]$. An efficiency function is added to correct the reduction of flame surface induced by the thickening operation [16, 17]. Charlette et al. [17] express this term as a sub-grid scale wrinkling factor, $\Xi_{\Delta}$, that measures the ratio between the total and the resolved flame surface. Thus, the balance equations for filtered species mass fractions $\widetilde{Y}_{k}$ are written as:

$$
\frac{\partial \bar{\rho} \widetilde{Y}_{k}}{\partial t}+\nabla \cdot\left(\bar{\rho} \widetilde{\mathbf{u}} \widetilde{Y}_{k}\right)=-\nabla \cdot\left(\Xi_{\Delta} \mathcal{F} \overline{\rho \mathbf{V}_{k}} \widetilde{Y}_{k}\right)+\frac{\Xi_{\Delta}}{\mathcal{F}} \dot{\omega}_{k}(\widetilde{Q})
$$

where $\rho$ is the density, $\mathbf{u}$ the velocity vector, $\mathbf{V}_{\mathbf{k}}$ the diffusion velocity of species $k$, expressed here using the Hirschfelder and Curtiss approximation $[45,46]$ and $\dot{\omega}_{k}$ the reaction rate of species $k$, estimated from Arrhenius law. Any quantity $\bar{Q}$ corresponds to the filtering of the Q-field, while $\widetilde{Q}=\overline{\rho Q} / \bar{\rho}$ denotes mass-weighted filtering. Similarly, the balance equation 
for the filtered total energy $\widetilde{E}$ is written:

$$
\begin{aligned}
\frac{\partial \bar{\rho} \widetilde{E}}{\partial t}+\nabla \cdot(\bar{\rho} \widetilde{\mathbf{u}} \widetilde{E})= & -\nabla \cdot\left[\widetilde{\mathbf{u}} \bar{P}-\widetilde{\mathbf{u}} \overline{\boldsymbol{\tau}}+\Xi_{\Delta} \mathcal{F}\left(\overline{\mathbf{q}}_{T}-\sum_{k=1}^{N} \overline{\rho \mathbf{V}_{k}} \widetilde{Y}_{k} \widetilde{h}_{s, k}\right)\right] \\
& +\frac{\Xi_{\Delta}}{\mathcal{F}} \dot{\omega}_{T}(\widetilde{Q})
\end{aligned}
$$

where $\bar{P}$ is the filtered pressure, $\widetilde{h}_{s, k}$ the sensible enthalpy of species $k, \bar{\tau}$ the viscous tensor, $\bar{q}_{T}$ the thermal flux due to temperature gradients modeled using a Fourier law and $\dot{\omega}_{T}$ the heat release rate. Equations $(1-2)$ propagate a flame front of thickness $\mathcal{F} \delta_{L}^{0}$ at the sub-grid scale turbulent velocity $S_{T}=$ $\Xi_{\Delta} S_{L}$, where $\delta_{L}^{0}$ is the laminar flame thickness.

\subsection{Dynamic wrinkling model}

Charlette et al. modeled the wrinkling factor $\Xi_{\Delta}$ by an algebraic expression derived assuming an equilibrium between turbulence motions and flame front wrinkling [17]:

$$
\Xi_{\Delta}=\left(1+\min \left[\max \left(\frac{\Delta}{\delta_{L}^{0}}-1,0\right), \Gamma_{\Delta}\left(\frac{\Delta}{\delta_{L}^{0}}, \frac{u_{\Delta}^{\prime}}{S_{L}}, R e_{\Delta}\right) \frac{u_{\Delta}^{\prime}}{S_{L}}\right]\right)^{\beta}
$$

where the efficiency function $\Gamma_{\Delta}$ measures the ability of vortices to effectively wrinkle the flame front, $u_{\Delta}^{\prime}$ and $R e_{\Delta}=u_{\Delta}^{\prime} \Delta / \nu$ are the sub-grid scale turbulence intensity and Reynolds number, respectively, $\nu$ being the fresh gas kinematic viscosity. $\beta$ is the model parameter to be specified. Note that the $(-1)$ contribution in Eq. (3) was introduced later [7] to recover Eq. (4) below in the limit of large turbulence intensities. In practice, Eq. (3) is often saturated and dominated by $\Delta / \delta_{L}^{0}$, reducing to [47]:

$$
\Xi_{\Delta}=\left(\frac{\Delta}{\delta_{L}^{0}}\right)^{\beta}
$$


corresponding to a fractal model with a flame surface of fractal dimension $D=\beta+2$ and an inner cut-off scale sets to the laminar flame thickness $\delta_{L}^{0}[24,48,49]$. The exponent $\beta$ is now determined dynamically equating flame surfaces when computed at filtered and test-filtered level (Germanolike identity) $[5,8,47,50]$ :

$$
\left\langle\overline{\Xi_{\Delta}|\nabla \widetilde{c}|}\right\rangle=\left\langle\Xi_{\gamma \Delta}|\nabla \widehat{\widetilde{c}}|\right\rangle
$$

where the hat symbol denotes a test-filtering operation. The effective filter size when combining two Gaussian filters of size $\Delta$ and $\widehat{\Delta}$, is $\gamma \Delta$, with

$$
\gamma=\sqrt{1+\left(\frac{\widehat{\Delta}}{\Delta}\right)^{2}}
$$

Symbol $\langle\cdot\rangle$ denotes an averaging operator [4] that may be the overall computational volume (global formulation) or a small local volume (local formulation). For the latter case and following $[47,50]$, the averaging operation is here replaced by a Gaussian filter $\Delta_{a v g}$, easier to implement on massively parallel solvers with unstructured meshes [39]. Combining Eqs (4) and (5) and assuming that $\beta$ is constant over the averaging domain $\langle\cdot\rangle$ give:

$$
\beta=\frac{\log (\langle\widehat{\nabla \widetilde{\widetilde{c}} \mid}\rangle /\langle|\nabla \widehat{\widetilde{c}}|\rangle)}{\log (\gamma)}
$$

However, strictly speaking, a thickened flame is not a filtered flame following the standard LES definitions and an equivalent filter size $\Delta$ should be specified to enter relations (4) and (6). As the inner cut-off scale is estimated 
to be the laminar flame thickness $\delta_{L}^{0}$ in Eq. (4), we relate here the outer cutoff scale, $\Delta$, to the resolved flame thickness, i.e. $\Delta=\mathcal{F} \delta_{L}^{0}$. Equation (4) becomes:

$$
\Xi_{\Delta}=\mathcal{F}^{\beta}
$$

Note that Wang et al. [7], deriving their dynamic model by comparing reaction rates, wrote $\Delta=\alpha \mathcal{F} \delta_{L}^{0}$, introducing a calibration factor $\alpha=2.2$ to recover $\beta=0$ and $\Xi_{\Delta}=1$ for planar laminar flames. Here, the model is derived from flame surfaces and this condition is automatically enforced by Eq. (7).

In the following, test and averaging filter sizes are constant and equal to $2.0 \Delta$ and $2.7 \Delta$ respectively. Figures 10 and 17 discussed later will confirm that results are not sensitive the test filter width. The influence of the averaging filter was not investigated here but its characteristics were chosen according to a previous study [9]. Increasing its width mainly reduces model parameter and wrinkling factor variances without affecting the overall results, at least when not too large.

\subsection{Wall correction for the global formulation}

The interaction of turbulence, flame and wall is a complex phenomenon, which is present in the majority of practical industrial systems. The objective of this work is not to develop a model for turbulent flame/wall interactions and a simple wall correction is introduced as a first step in the global saturated dynamic formulation. Walls limit flame wrinkling and act as a sink term for the flame surface density $\Sigma$ or the wrinkling factor $\Xi_{\Delta}[45,51,52]$. Thus, while the local formulation automatically predicts low wrinkling factors $\Xi_{\Delta}$ near walls because of reduced resolved flame wrinkling, the global 
formulation, assuming uniform model parameter $\beta$ and wrinkling factor $\Xi_{\Delta}$ over the computational domain, needs correction. In this latter case, a simple ad hoc damping function is implemented:

$$
\Xi_{\Delta}=\left[1-\exp \left(\frac{-d_{w}}{0.5 \Delta}\right)\right]\left[\left(\frac{\Delta}{\delta_{L}^{0}}\right)^{\beta}-1\right]+1
$$

where $\Delta$ is the combustion filter size and $d_{w}$ is the distance to the closest wall. Such an expression affects the wrinkling factor for $d_{w}<2 \Delta$, but because of the exponential, the influence is initially small: the wrinkling factor is equal to $87 \%$ of its initial value for $d_{w}=\Delta$, then goes to unity at wall. This model is rough and is designed to reduce sub-grid scale flame wrinkles because of mechanical constraints without taking into account explicitly heat losses. Anyway, the importance of this correction is more conceptual (wrinkling factors are not expected to be large in the vicinity of walls) than practical: its influence on statistics is negligible (not shown for brevity). A refined lawof-the-walls model for combustion, compatible with the proposed modeling approach, is left for future works.

\section{The Preccinsta burner}

\subsection{Experimental configuration}

The burner configuration investigated by Meier et al. [1] derives from an industrial gas turbine designed by Turbomeca. This configuration, representative of a realistic combustor, has been widely studied to validate combustion models, reduced chemical mechanisms or numerical methods [3642]. The combustor, displayed in Fig. 1, comprises four main parts: 1) the 
plenum, where ambient air is injected through one large hole; 2) the injector, where the air flow is swirled by twelve radial veins and mixed with pure methane injected through the swirler blades; 3) the combustion chamber of dimensions $114 \mathrm{~mm} \times 85 \mathrm{~mm} \times 85 \mathrm{~mm}$ equipped with $1.5 \mathrm{~mm}$ thick quartz walls to enable optical measurements; 4) the converging tube that connects the combustion chamber to the atmosphere. Three different regimes, summarized in Table 1, were studied experimentally. Only cases at equivalence ratio $\phi=0.70$ (case 1 ) and $\phi=0.83$ (case $2 \mathrm{a}$ ) are addressed herein.

Laser Raman scattering measurements are available for concentration of the major species $\left(\mathrm{CH}_{4}, \mathrm{H}_{2} \mathrm{O}, \mathrm{O}_{2}, \mathrm{CO}_{2}, \mathrm{CO}, \mathrm{N}_{2}, \mathrm{H}_{2}\right)$ and temperature in vertical planes at eight different sections downstream of the injector $(h=$ $6,10,15,20,30,40,60,80 \mathrm{~mm}$, where $h=0 \mathrm{~mm}$ corresponds to the exit plane of the nozzle). The systematic and statistical uncertainties are less than $4 \%$ and $2.5 \%$ respectively for temperature and less than $5 \%$ and $7 \%$ respectively for all species except for $\mathrm{CO}$ and $\mathrm{H}_{2}$ for which statistical uncertainty is between $20 \%$ and $50 \%$.

Laser Doppler Velocimetry (LDV) measurements of the velocity field were also performed in sections located at five different axial positions $(h=$ $1.5,5,15,25,35 \mathrm{~mm})$. However, these measurements were carried out at a global equivalence ratio of $\phi=0.75$ (case $2 \mathrm{~b}$ ) and not $\phi=0.83$ (case 2a) unfortunately. Thereby, comparisons between numerical results and experimental data must be accomplished with care.

\subsection{Numerical setup}

Instead of injecting fuel and oxidizer separately, a perfectly methane/air mixture is directly injected in the plenum inlet. This perfect mixing assump- 


\begin{tabular}{llll}
\hline Experimental case & 1 & $2 \mathrm{a}$ & $2 \mathrm{~b}$ \\
\hline Air flow rate (g/min) & 734.2 & 734.2 & 734.2 \\
$\mathrm{CH}_{4}$ flow rate (g/min) & 30.0 & 35.9 & 32.3 \\
Thermal power $(\mathrm{kW})$ & 25.1 & 30.0 & 27.0 \\
Equivalence ratio (-) & 0.70 & 0.83 & 0.75 \\
Experimental behavior & Unstable & Stable & Stable \\
Measurements & LDV+Raman & Raman & LDV \\
\hline
\end{tabular}

Table 1: Details of the experimental cases.

tion was made because works in the literature showed good agreement at least for the stable regime [36-39, 42].

The AVBP compressible solver developed at CERFACS and IFPEN is employed to run the simulations [53]. An overview of the computational domain is illustrated in Fig. 1. The plenum and atmospheric inlets were prescribed using the Navier-Stokes Characteristic Boundary conditions (NSCBC) [54] to ensure a physical representation of the acoustic wave propagation and reflection. The burner is fed steadily with a mixture of methane and air at an equivalence ratio of $0.83 / 0.70$, and the total mass flow rate is $12.9 \mathrm{~g} / \mathrm{s}$ at a temperature of $320 \mathrm{~K}$. For the inlet of the atmosphere, a co-flow of $N_{2}$ is injected with low velocity $(0.5 \mathrm{~m} / \mathrm{s})$ at the temperature of the burnt gases to mimic entrainment and avoid any unphysical recombination between burnt and fresh air at the chamber exit. The outer atmosphere boundary is also specified using NSCBC. Adiabatic and no-slip conditions are imposed at walls. These choices were based on the work by Franzelli et al. [42].

The third-order finite element TTGC scheme [55] is used. Sub-grid 
stresses are described by the WALE model [56]. A two-step reduced chemical mechanism for the methane oxidation is considered [57]:

$$
\begin{aligned}
& \mathrm{CH}_{4}+1.5 \mathrm{O}_{2} \longrightarrow \mathrm{CO}+2 \mathrm{H}_{2} \mathrm{O} \\
& \mathrm{CO}+0.5 \mathrm{O}_{2} \longleftrightarrow \mathrm{CO} 2
\end{aligned}
$$

The corresponding reaction rates are modeled using Arrhenius laws:

$$
\begin{aligned}
& q_{1}=A_{1} \exp \left(\frac{-E_{a 1}}{R T}\right)\left(\frac{\rho Y_{C H_{4}}}{W_{C H_{4}}}\right)^{n_{C H_{4}}^{1}}\left(\frac{\rho Y_{O_{2}}}{W_{O_{2}}}\right)^{n_{O 2}^{1}} \\
& q_{2}=A_{2} \exp \left(\frac{-E_{a 2}}{R T}\right)\left[\left(\frac{\rho Y_{C O}}{W_{C O}}\right)^{n_{C O}^{2}}\left(\frac{\rho Y_{O_{2}}}{W_{O_{2}}}\right)^{n_{O 2}^{2}}-\frac{1}{K_{e}}\left(\frac{\rho Y_{C O_{2}}}{W_{C O_{2}}}\right)^{n_{C O_{2}}^{2}}\right]
\end{aligned}
$$

where pre-exponential factors, activation energies and model exponents are summarized on Table 2. $K_{e}$ is the equilibrium constant for the second reaction. The corresponding laminar flame properties are listed in Table 3.

\begin{tabular}{|c|l|c|c|}
\hline Reaction & Coefficients & $A_{n}$ & $E_{a n}$ \\
\hline 1 & $n_{C H_{4}}^{1}=0.9 n_{\mathrm{O}_{2}}^{1}=1.1$ & $2.0 \times 10^{15}$ & $3.5 \times 10^{4}$ \\
\hline 2 & $n_{\mathrm{CO}}^{2}=1.0 n_{\mathrm{O}_{2}}^{2}=0.5$ & $2.0 \times 10^{9}$ & $1.2 \times 10^{4}$ \\
& $n_{\mathrm{CO}_{2}}^{2}=1.0$ & & \\
\hline
\end{tabular}

Table 2: Two-step reduced chemical mechanism for $\mathrm{CH}_{4}-\mathrm{Air}$. Coefficients for reaction rates [57]. Activation energies are in cal $/ \mathrm{mol}$ and pre-exponential constants in cgs units.

The full geometry is meshed as shown in Fig. 1. The mesh is unstructured and contains about $21 \mathrm{M}$ cells and $3.7 \mathrm{M}$ nodes. The typical cell size in the reaction zone is $\Delta_{x} \approx 0.5 \mathrm{~mm}$. The thickening factor $\mathcal{F}$ is chosen to resolve the flame front with at least $n$ cells in the simulation, i.e. such as $\mathcal{F} \delta_{L}^{0} \geq n \Delta_{x}$. 


\begin{tabular}{llll}
\hline$\phi$ & $S_{L}[\mathrm{~m} / \mathrm{s}]$ & $\delta_{L}^{0}[\mathrm{~mm}]$ & $T_{b}[\mathrm{~K}]$ \\
\hline 0.83 & 0.30 & 0.40 & 2058 \\
0.70 & 0.20 & 0.55 & 1846 \\
\hline
\end{tabular}

Table 3: Laminar flame properties for different mixtures at $P_{a t m}$ and $T_{a t m}$. $S_{L}, \delta_{L}^{0}$ and $T_{b}$ stand for the laminar flame speed, the laminar flame thickness based on the temperature gradient and the burnt gas temperature, respectively.

Here, according to Table $3, \Delta_{x} / \delta_{L}^{0}$ goes from $0.9(\phi=0.70)$ to $1.25(\phi=0.83)$ while usually $n=4-5$ with AVBP. The thickening factor is then set to $\mathcal{F}=5$.

\section{Data processing}

\subsection{Flow field statistics}

LES of compressible reactive flows computes Favre (mass-weighted) filtered quantities $\widetilde{Q}$. "Quasi-Reynolds" average over a time period $\tau$ and the corresponding resolved variance are defined as:

$$
\begin{aligned}
{[\widetilde{Q}] } & =\frac{1}{\tau} \int_{0}^{\tau} \widetilde{Q}(\mathbf{x}, t) d t \\
{[\widetilde{Q}]_{r m s} } & =\left(\left[\widetilde{Q}^{2}\right]-[\widetilde{Q}]^{2}\right)^{1 / 2}
\end{aligned}
$$


Favre average and resolved variance can be estimated as [58]:

$$
\begin{aligned}
\{\widetilde{Q}\} & =\frac{[\bar{\rho} \widetilde{Q}]}{[\bar{\rho}]} \\
\{\widetilde{Q}\}_{r m s} & =\frac{1}{[\bar{\rho}]}\left(\left[\bar{\rho}(\widetilde{Q})^{2}\right]-\frac{[\bar{\rho} \widetilde{Q}]^{2}}{[\bar{\rho}]}\right)^{1 / 2}
\end{aligned}
$$

"Quasi-Reynolds" averages are widely retained because most diagnostic

techniques provide unweighted filtered quantities and $\left[\widetilde{Y}_{k}\right]$ is closer to experimental results than $\left\{\tilde{Y}_{k}\right\}[58]$. Fortunately, for the PRECCINSTA burner both Reynolds and Favre statistics are available.

Statistics are collected over $20 \mathrm{~ms}$. This duration may be compared to the turn-over time of the swirl motion, which is about $2.0 \mathrm{~ms}$. The total wall clock time for both convergence and statistics are around of 50h on 2048 cores of an IBM BlueGene/P machine for the stable case and twice as long for the unstable one.

\subsection{Model parameter statistics}

For the local formulation, the mean combustion model parameter, $\bar{\beta}(t)$ and its fluctuation, $\beta^{\prime}(t)$ are computed using the following definitions:

$$
\begin{aligned}
& \bar{\beta}(t)=\frac{\int_{\mathcal{V}_{f}} \beta(x, y, z, t) d \mathcal{V}}{\int_{\mathcal{V}_{f}} d \mathcal{V}} \\
& \beta^{\prime}(t)=\sqrt{\overline{\beta^{2}}(t)-(\bar{\beta}(t))^{2}}
\end{aligned}
$$

where $\mathcal{V}_{f}$ denotes the instantaneous resolved flame volume defined as $\varepsilon \leq$ $\widetilde{c} \leq 1-\varepsilon$, for $\varepsilon=0.05$. 


\subsection{Flame surfaces statistics}

Resolved flame surfaces at the filter $(\Delta)$ and test-filtered $(\widehat{\Delta})$ scales can be estimated as:

$$
\begin{aligned}
& S_{r}=\int_{\mathcal{V}}|\nabla \widetilde{c}| d \mathcal{V} \\
& \widehat{S}_{r}=\int_{\mathcal{V}}|\nabla \widehat{\widetilde{c}}| d \mathcal{V}
\end{aligned}
$$

where $\mathcal{V}$ is the computational volume.

\section{Simulations results and discussions}

Results are now discussed for the quiet and pulsating flames. Table 4 resumes the simulated cases.

\subsection{The quiet flame $-\phi=0.83$}

All simulations of Case $2 \mathrm{a}(\phi=0.83)$ reproduce a stable flame stabilized at the nozzle exit in agreement with experiments. When using the local approach, the model parameter is low near the nozzle and increases downstream as the flame is progressively wrinkled by turbulence (see Fig. 2). Therefore, a constant/global model parameter may overestimate the front wrinkling factor during the early flame development and underestimate it when flame/turbulence equilibrium is reached. These observations emphasize the advantages of the local formulation.

Figure 3 compares snapshots of the heat release field for simulations using global and local approaches. Note that in the global case, the heat release is uniform all over the flame. On the other hand, in the local case, the heat release increases in regions where the flame is more wrinkled. The constant 


\begin{tabular}{llll}
\hline Simulation & Model parameter & $\phi$ & $\begin{array}{l}\text { Numerical } \\
\text { behavior }\end{array}$ \\
\hline$C_{0.83}$ & $\beta=0.5$ & 0.83 & Stable \\
$G_{0.83}$ & Global: $\beta(t)$ & 0.83 & Stable \\
$L_{0.83}$ & Local: $\beta(\mathbf{x}, t)$ & 0.83 & Stable \\
$C_{0.70}$ & $\beta=0.5$ & 0.70 & Stable \\
$G_{0.70}$ & Global: $\beta(t)$ & 0.70 & Unstable \\
$L_{0.70}$ & Local: $\beta(\mathbf{x}, t)$ & 0.70 & Unstable \\
\hline
\end{tabular}

Table 4: Summary of simulated cases. $C, G$ and $L$ stand for constant, global and local formulations, respectively. The subscript of $X_{\alpha}$ denotes the equivalence ratio. Simulations using the constant model assumed $\beta=0.5$, according to Charlette et al. [17]. Filter, test-filter and averaging filter widths are set to $\Delta=\mathcal{F} \delta_{L}^{0}, \widehat{\Delta}=2.0 \Delta$ and $\Delta_{\text {avg }}=2.7 \Delta$, respectively, following Volpiani et al. [9], where $\mathcal{F}$ is the flame thickening factor and $\delta_{L}^{0}$ the laminar flame thickness. 
model parameter (not shown) gives the same distribution than the global dynamic model.

Figure 4 compares Reynolds (Eqs 14 - 15) and Favre (Eqs 16 - 17) mean and RMS methane profiles at five different sections in the chamber for the local approach $\left(L_{0.83}\right)$. Favre filtered quantities agree with Favre measurements [58]. Interestingly, quasi-Reynolds filtered quantities compare to Reynolds measurements as well. Even though Veynante and Knikker [58] showed that $\left[\widetilde{Y}_{k}\right]$ is not a good approximation of $\left[Y_{k}\right]$, at least for infinitely thin flame fronts, most works retain this approximation that seems acceptable for both mean and RMS quantities. In the following, only Favre averages and variances will be displayed.

Figure 5 compares Favre mean and resolved RMS carbon dioxide mass fraction profiles at five different sections in the combustion chamber. Local and global formulations of the TFLES dynamic model give very close statistics in agreement with experimental data, validating the present combustion model. The description of species fluctuations is similar with both formulations. The discrepancies observed on $h=20 \mathrm{~mm}$ profiles might be due to insufficiently converged statistics, missing low frequency motions of the inner recirculation zone and/or the flame tip. Using a constant model parameter $\beta=0.5$ (as recommended in [17]) gives similar results as the global approach for which the model parameter is found to slightly oscillate around this value. Anyway, mean and RMS profiles are probably not sufficient to fully assess model formulations and additional experimental data characterizing the flame dynamics are mandatory.

Mean and resolved RMS temperature profiles are displayed in Fig. 6. 
Once again, the experiment is well reproduced by simulations. The overestimation of temperature near walls at $h=6$ and $10 \mathrm{~mm}$, at the outer recirculation zone, are due to the adiabaticity hypothesis: heat losses and radiation effects are neglected in the present simulations. Similar temperature overestimation is observed in other studies conducted under the same assumption [39].

Mean $C O$ mass fraction profiles are compared to experimental points in Fig. 7. For this species, the statistical uncertainty reaches $50 \%$ and is also plotted. Even if some differences appear close to the injector at the outer recirculation zone, the results are very satisfactory for a reduced mechanism that takes into consideration only 2 reactions and 6 species. The overestimation of mean $C O$ mass fraction profiles close to the injector at $R=20 \mathrm{~mm}$ are probably due to the overestimation of temperature in the same region.

Overall, even for simulation using a constant model parameter, LES statistical properties are in line with measurements. However, note that for case $C_{0.83}$, the model parameter was known a priori from simulation $G_{0.83}$ (Fig. 8). Still, the global formulation has the advantage to adapt automatically the model parameter without the need to adjust it by hand case-by-case, giving a decisive advantage to the dynamic formalism. Figure 8 displays the temporal evolution of the global parameter that oscillates around 0.5. The mean and RMS model parameter from simulation $L_{0.83}$ are also plotted. One may note that the mean parameter value predicted by the local formalism, about $\beta=0.60$, is slightly larger than the one found with the global model $(\beta \approx 0.52)$ while statistics are very similar (Figs $5-7)$. This finding might be due to the thresholding introduced in definition (18) that removes the low 
values of the resolved progress variable gradients at fresh $(\widetilde{c}<\varepsilon)$ and burnt $(\widetilde{c}>1-\varepsilon)$ gas limits, while these values enter the computation of the global parameter.

Assuming a fractal behavior of the flame surface (Eq. 4) gives:

$$
\frac{\Xi_{\Delta}}{\Xi_{\gamma \Delta}}=\frac{\widehat{S}_{r}}{S_{r}}=\left(\frac{\gamma \Delta}{\Delta}\right)^{-\beta}
$$

Figure 9 shows how the flame surface changes when a test-filter is applied. According to Eq. (22), the normalized filtered flame surface is then expected to be a straight line of slope $-\beta$ when plotted as a function of the filter width ratio in log-log scales, as confirmed by Fig. 10, where the test filter width is varied from $\Delta$ to $4.0 \Delta$ taking into consideration the entire domain, upstream $(h<15 \mathrm{~mm})$ and downstream $(h>15 \mathrm{~mm})$ half of the flame where $h$ is the downstream location. This figure also evidences the relevance and the robustness of the dynamic formulation as the model parameter $\beta$ is only very marginally dependent on $\widehat{\Delta}$, at least when not too large. In fact, plots display a slight curvature but its impact on $\beta$ values is negligible. The mean slope of the curve for the overall domain gives $\beta=0.58$. Concerning the local analysis, we found $\beta=0.42$ for the first half and 0.68 for the second half of the flame. These results are in agreement with Fig. 11 that displays the distribution of $\beta$ for the same zones and timestep.

Figure 11 shows that the local parameter exceeds unity in some limited regions. Two explanations may be suggested to explain this a priori surprising finding: ( $i$ ) the model is written here in terms of "generalized" surfaces (i.e. $|\nabla \widetilde{c}|$ ) and not surfaces corresponding to a given value $c^{*}$ of the filtered progress variable (i.e. $\widetilde{c}=c^{*}$ ); (ii) in highly wrinkled resolved flame re- 
gions or when flame fronts interact, $|\nabla \widehat{\widetilde{c}}|$ may takes low values, increasing the model parameter $\beta$ (Eq. 7). This point needs further analysis but these locally large $\beta$ values do not induce numerical problems nor affect overall results here. Note, however, that Mouriaux et al. [29] faced recently practical difficulties for internal combustion engines where ratios $\Delta / \delta_{L}^{0}$ are large, leading to large wrinkling factor values, and introduced model corrections.

\subsection{The pulsating flame $-\phi=0.70$}

Figure 12 displays the temporal evolution of the total heat release per unit volume for the three numerical simulations performed at this operating point (Table 4). While the total heat release oscillates due to strong flame movements when using the dynamic approach, a stable regime is observed with a constant model parameter. Assuming perfect reactant mixing, Franzelli et al. [42] also found a quiet flame with a non-dynamic model. In Fig. 13, a zoom on the heat release signal is plotted with the temporal evolution of the pressure fluctuation in the combustion chamber, $P_{c}$, and in the plenum, $P_{p}$. Heat release and pressure $P_{c}$ oscillate at the same frequency, suggesting that the instability is fed by a flame/acoustic coupling. Moreover, chamber and plenum pressure fluctuations oscillates practically in phase opposition, in agreement with mechanisms identified in other studies [42].

The predicted frequency is about $500 \mathrm{~Hz}$ while $300 \mathrm{~Hz}$ is observed in the experiment. Franzelli et al. [42] found $400 \mathrm{~Hz}$, when injecting the reactants separately. These discrepancies are probably linked to the injection impedance, which was not characterized in the experiment and arbitrarily imposed in LES. Note also that a simple turbulent velocity profile is imposed at the plenum inlet and walls are assumed adiabatic in simulations, which 
may differ from the actual experimental conditions. Further investigations are required to confirm these findings but our goal is also to point out that a relatively minor change in the model may induce large variation in the unsteady flow behavior. To our feeling, the description of mixing in Franzelli et al. [42] or the dynamic formalism in the present results introduces a degree of freedom in reaction rate expressions, making them more sensitive to external perturbations, possibly promoting the development of an unstable mode. These modes are also observed for the stable case but with reduced amplitudes.

The cycle of the self-sustained oscillation is visualized in Fig. 14 displaying the instantaneous $\widetilde{c}=0.5$ isosurface colored by $\beta$ and the $\beta$ probability density function for eight phases of the plenum pressure $P_{p}$ fluctuation, referred as ph1 to ph8 in Ref. [1] and Fig. 13. During ph1, the plenum pressure is minimal. The flame is fully developed at ph2. At this point, the combustion intensity is maximal. The high pressure inside the combustion chamber forces the flame to move upstream. In ph3, the flame starts to disintegrate and is pushed back inside the injector. The flame is located practically inside the nozzle during $p h 4$ and $p h 5$, when the plenum pressure is maximal. During $p h 6$, the flame starts its expansion and re-enters the combustion chamber, where pressure is minimal. In $p h 7$ and $p h 8$, the flame moves downstream reinitiating the cycle. For the global approach the model parameter is minimal (maximal) when the flame is shorter (longer).

Favre mean and resolved RMS mass fraction profiles for the three simulations studied are compared to experimental results in Fig. 15. When using the dynamic (local or global) model, mean and RMS profiles of $\mathrm{CH}_{4}$ 
provide similar levels of agreement with experiments. On the other hand, numerical results are deteriorated when the model parameter is fixed. This finding is not surprising, because as already mentioned in [42] and confirmed by Fig. 12, the unstable mode is not recovered when imposing a constant model parameter. Figure 16 displays the mean and RMS axial velocity at five sections downstream of the injector. The negative velocity characterizing the inner recirculation zone reaches approximately $20 \mathrm{~m} / \mathrm{s}$ at $h=1.5 \mathrm{~mm}$ and is recovered numerically. The RMS of the axial velocity was not measured experimentally but is almost of the same order of the mean value, indicating a strong activity inside the combustion chamber.

One of the most interesting points is that the fractal dimension $(D=$ $\beta+2)$ is actually not constant and depends on time within a period of the instability. This is evidenced plotting the normalized filtered flame surfaces as a function of the ratio of filters, $\gamma$, in $\log -\log$ scales, considering the entire domain as done in the previous section. The slope and accordingly $\beta$ values depend on the phase within the oscillation, as shown in Fig. 17. The straight lines in this figure confirms the robustness of the dynamic formulation as, once again, the model parameter is not sensitive to the test filter width.

These results are also confirmed by Fig. 18 that displays the temporal evolution of the mean model parameter, $\bar{\beta}$ (Eq. 18), and the total heat release for simulation using the local dynamic model $\left(L_{0.70}\right)$. In contrast to the stable flame, the $\bar{\beta}$ oscillates strongly between 0.4 and 1.1 . Moreover, the model parameter oscillates in phase with the heat release, meaning that $\beta$ is maximum (minimum) when the flame is expanded (compressed). Similar results can be found for the global case $\left(G_{0.70}\right)$, as confirmed in Fig. 19 . 
Note that differences between mean parameter values displayed in Figs. 17 and 18 are due to the fact that the slope computation is based on one single snapshot and Eqs (20-22), while the mean model parameter in Fig. 18 is estimated from Eq. (18) using a threshold.

\subsection{Mesh convergence for the pulsating flame}

Even though recent experimental works $[59,60]$ treated the same combustor burning perfectly premixed methane-air flames and investigated the thermo-acoustic coupling, data are not yet available for comparison to the unstable case simulated herein. Any change in the configuration (geometry, boundary conditions, etc) may damp or promote self-excited oscillations of the burner [61]. Therefore, the model validation requires to check whether the oscillations reproduce the physics or are artificially introduced by the dynamic formalism. The local dynamic model was tested using a finer mesh with $65 \mathrm{M}$ cells $/ 11 \mathrm{M}$ nodes and a thickening factor $\mathcal{F}=3$ reducing the impact of the LES model. The CPU time for computation using the fine mesh is approximately 4 times longer than the standard mesh (21M cells/3.7M nodes). In order to have the same physical time, 8192 cores were used to run the simulation (instead of 2024) on the same IBM BlueGene/P machine. Figure 20 displays the temporal evolution of the total heat release using the local dynamic model for both meshes. The strong oscillation is also present in the simulation using the fine grid. A slight phase shift between coarse and fine grid simulations is observed during the instability development but instantaneous LES signals cannot be directly compared: only the comparison of statistics, such as phase averages during the well-established instability cycle, is relevant [14]. Time-shifting signals to superimpose the last periods 
(not displayed here) shows that pulsation frequencies are identical, validating previous results. This conclusion is also confirmed by mean and RMS profiles, as shown in Fig. 21. Instantaneous field of $\widetilde{c}=0.5$ iso-surface colored by the $\beta$ parameter for simulation using the fine mesh for 8 different phases can be found as supplementary material.

\section{Conclusion}

In this work, the global and local dynamic formulations for the flame wrinkling factor were combined with the Thickened Flame (TFLES) model and simulations of the semi-industrial PRECCINSTA burner studied experimentally by Meier et al. [1] were performed for the stable and unstable cases. The global formulation predicts a time-dependent model exponent that remains at a level close to 0.5 for the stable flame and oscillates strongly around 0.8 for the pulsating flame. Moreover, the global saturated model was corrected by applying a wrinkling factor damping near the walls to take into account flame/wall interactions. The local formulation adapts the parameter locally based on the gradients of the resolved progress variable all over the domain and does not need this modification. The usual non-dynamic formulation with an appropriate constant, to be set by trial and error, is sufficient to capture flow and combustion time averaged and RMS fields with good accuracy (both in terms of Favre and quasi-Reynolds quantities) in the stable case. However, the self-excited modes of the pulsating flame are captured only with the dynamic model. The fractal dimension of the unstable flame is found to vary locally and depends on the phase within the period of oscillation. While Franzelli et al. [42] suggested that methane/air mixing 
could be linked to the flame pulsation, the details of the exact mechanism controlling the instability itself has not been identified yet. We conclude that dynamic models may have an effect on the prediction of combustion instabilities. A simulation combining the description of the fuel / air mixing with the dynamic formalism would be an interesting complementary test. Unfortunately, it cannot be conducted in a near future because the dynamic model has been developed and validated only for premixed combustion and must be first extended to partially premixed and non-premixed regimes. Also, such a simulation needs large computational ressources: the fuel is injected through small holes in the swirler vanes requiring a very refined mesh in this region. Anyway, the unsteady behavior of the dynamic model will be analyzed in details in a near future to fully understand our findings.

\section{Acknowledgments}

This work was performed using HPC resources of IDRIS under the allocation 2015-90164 made by GENCI (Grand Equipement National de Calcul Intensif). The authors would like to thank Prof. W. Meier and Dr. B. Franzelli for fruitful discussions.

\section{References}

[1] W. Meier, P. Weigand, X. Duan, R. Giezendanner-Thoben, Detailed characterization of the dynamics of thermoacoustic pulsations in a lean premixed swirl flame, Combust. Flame 150 (2007) 2-26.

[2] M. Germano, U. Piomelli, P. Moin, W. H. Cabot, A dynamic subgrid- 
scale eddy viscosity model, Phys. Fluids A: Fluid Dynamics (1989-1993) 3 (1991) 1760-1765.

[3] H. G. Im, T. S. Lund, J. H. Ferziger, Large eddy simulation of turbulent front propagation with dynamic subgrid models, Phys. Fluids 9 (1997) $3826-3833$.

[4] F. Charlette, C. Meneveau, D. Veynante, A power-law flame wrinkling model for les of premixed turbulent combustion Part II: Dynamic formulation, Combust. Flame 131 (2002) 181-197.

[5] G. Wang, M. Boileau, D. Veynante, K. Truffin, Large eddy simulation of a growing turbulent premixed flame kernel using a dynamic flame surface density model, Combust. Flame 159 (2012) 2742-2754.

[6] E. Knudsen, H. Pitsch, A dynamic model for the turbulent burning velocity for large eddy simulation of premixed combustion, Combust. Flame 154 (2008) 740-760.

[7] G. Wang, M. Boileau, D. Veynante, Implementation of a dynamic thickened flame model for large eddy simulations of turbulent premixed combustion, Combust. Flame 158 (2011) 2199-2213.

[8] T. Schmitt, M. Boileau, D. Veynante, Flame wrinkling factor dynamic modeling for large eddy simulations of turbulent premixed combustion, Flow Turb. Combust. 94 (2015) 199-217.

[9] P. Volpiani, T. Schmitt, D. Veynante, A posteriori tests of a dynamic thickened flame model for large eddy simulations of turbulent premixed combustion, Combust. Flame 174 (2016) 166 - 178. 
[10] T. Schmitt, A. Sadiki, B. Fiorina, D. Veynante, Impact of dynamic wrinkling model on the prediction accuracy using the f-tacles combustion model in swirling premixed turbulent flames, Proc. Combust. Inst. 34 (2013) 1261-1268.

[11] R. Mercier, T. Schmitt, D. Veynante, B. Fiorina, The influence of combustion SGS submodels on the resolved flame propagation. application to the LES of the Cambridge stratified flames, Proc. Combust. Inst. 35 (2015) 1259-1267.

[12] S. S. Ibrahim, S. R. Gubba, A. R. Masri, W. Malalasekera, Calculations of explosion deflagrating flames using a dynamic flame surface density model, J. Loss Prevention in the Process Industries 22 (2009) 258-264.

[13] S. R. Gubba, S. S. Ibrahim, W. Malalasekera, A. R. Masri, Measurements and les calculations of turbulent premixed flame propagation past repeated obstacles, Combust. Flame 158 (2011) 2465-2481.

[14] S. B. Pope, Ten questions concerning the large-eddy simulation of turbulent flows, New J. Phys. 6 (2004) 35.

[15] M. Boger, D. Veynante, H. Boughanem, A. Trouvé, Direct numerical simulation analysis of flame surface density concept for large eddy simulation of turbulent premixed combustion, in: Symp. (Int.) Combust., volume 27, Elsevier, pp. 917-925.

[16] O. Colin, F. Ducros, D. Veynante, T. Poinsot, A thickened flame model for large eddy simulations of turbulent premixed combustion, Phys. Fluids 12 (2000) 1843-1863. 
[17] F. Charlette, C. Meneveau, D. Veynante, A power-law flame wrinkling model for les of premixed turbulent combustion Part I: non-dynamic formulation and initial tests, Combust. Flame 131 (2002) 159-180.

[18] B. Fiorina, R. Vicquelin, P. Auzillon, N. Darabiha, O. Gicquel, D. Veynante, A filtered tabulated chemistry model for les of premixed combustion, Combust. Flame 157 (2010) 465-475.

[19] H. Pitsch, Large-eddy simulation of turbulent combustion, Annu. Rev. Fluid Mech. 38 (2006) 453-482.

[20] Y.-C. Chen, N. Peters, G. Schneemann, N. Wruck, U. Renz, M. S. Mansour, The detailed flame structure of highly stretched turbulent premixed methane-air flames, Combust. Flame 107 (1996) 223-IN2.

[21] B. Renou, Contribution à l'étude de la propagation d'une flamme de prémélange instationnaire dans un écoulement turbulent. Influence du nombre de Lewis, Ph.D. thesis, Université de Rouen, 1999.

[22] R. Knikker, D. Veynante, C. Meneveau, A priori testing of a similarity model for large eddysimulations of turbulent premixed combustion, Proc. Combust. Inst. 29 (2002) 2105-2111.

[23] R. Knikker, D. Veynante, C. Meneveau, A dynamic flame surface density model for large eddy simulation of turbulent premixed combustion, Phys. Fluids 16 (2004) L91-L94.

[24] F. Gouldin, An application of fractals to modeling premixed turbulent flames, Combust. Flame 68 (1987) 249-266. 
[25] Ö. L. Gülder, Turbulent premixed combustion modelling using fractal geometry, in: Symp. (Int.) Combust., volume 23, Elsevier, pp. 835-842.

[26] J. Bardina, J. Ferziger, W. Reynolds, Improved subgrid scale models for large eddy simulation, AIAA Paper No. 801357 (1980).

[27] C. Schneider, A. Dreizler, J. Janicka, Fluid dynamical analysis of atmospheric reacting and isothermal swirling flows, Flow Turb. Combust. 74 (2005) 103-127.

[28] M. Gregor, F. Seffrin, F. Fuest, D. Geyer, A. Dreizler, Multi-scalar measurements in a premixed swirl burner using 1D Raman/Rayleigh scattering, Proc. Combust. Inst. 32 (2009) 1739-1746.

[29] S. Mouriaux, O. Colin, D. Veynante, Adaptation of a dynamic wrinkling model to an engine configuration, Proc. Combust. Inst. 36 (2016).

[30] J. Réveillon, L. Vervisch, Subgrid-scale turbulent micromixing: Dynamic approach, AIAA journal 36 (1998) 336-341.

[31] C. D. Pierce, P. Moin, A dynamic model for subgrid-scale variance and dissipation rate of a conserved scalar, Phys. Fluids 10 (1998) 3041-3044.

[32] C. D. Pierce, P. Moin, Progress-variable approach for large-eddy simulation of non-premixed turbulent combustion, J. Fluid Mech. 504 (2004) 73-97.

[33] G. Balarac, H. Pitsch, V. Raman, Development of a dynamic model for the subfilter scalar variance using the concept of optimal estimators, Phys. Fluids 20 (2008) 035114. 
[34] C. M. Kaul, V. Raman, E. Knudsen, E. S. Richardson, J. H. Chen, Large eddy simulation of a lifted ethylene flame using a dynamic nonequilibrium model for subfilter scalar variance and dissipation rate, Proc. Combust. Inst. 34 (2013) 1289-1297.

[35] C. Dem, M. Stöhr, C. M. Arndt, A. M. Steinberg, W. Meier, Experimental study of turbulence-chemistry interactions in perfectly and partially premixed confined swirl flames, Zeitschrift für Physikalische Chemie 229 (2015) 569-595.

[36] S. Roux, G. Lartigue, T. Poinsot, U. Meier, C. Bérat, Studies of mean and unsteady flow in a swirled combustor using experiments, acoustic analysis, and large eddy simulations, Combust. Flame 141 (2005) 40-54.

[37] J. Galpin, A. Naudin, L. Vervisch, C. Angelberger, O. Colin, P. Domingo, Large-eddy simulation of a fuel-lean premixed turbulent swirl-burner, Combust. Flame 155 (2008) 247-266.

[38] V. Moureau, P. Minot, H. Pitsch, C. Bérat, A ghost-fluid method for large-eddy simulations of premixed combustion in complex geometries, J. Comput. Phys. 221 (2007) 600-614.

[39] V. Moureau, P. Domingo, L. Vervisch, From large-eddy simulation to direct numerical simulation of a lean premixed swirl flame: Filtered laminar flame-pdf modeling, Combust. Flame 158 (2011) 1340-1357.

[40] P. Wang, N. Platova, J. Fröhlich, U. Maas, Large eddy simulation of the PRECCINSTA burner, Int. J. Heat Mass Trans. 70 (2014) 486-495. 
[41] P. Wang, J. Frhlich, U. Maas, Z. xia He, C. jun Wang, A detailed comparison of two sub-grid scale combustion models via large eddy simulation of the PRECCINSTA gas turbine model combustor, Combust. Flame 164 (2016) $329-345$.

[42] B. Franzelli, E. Riber, L. Y. Gicquel, T. Poinsot, Large eddy simulation of combustion instabilities in a lean partially premixed swirled flame, Combust. Flame 159 (2012) 621-637.

[43] T. Butler, P. O'Rourke, A numerical method for two dimensional unsteady reacting flows, in: Symp. (Int.) Combust., volume 16, Elsevier, pp. $1503-1515$.

[44] P. O'Rourke, F. Bracco, Two scaling transformations for the numerical computation of multidimensional unsteady laminar flames, J. Comput. Phys. 33 (1979) 185-203.

[45] T. Poinsot, D. Veynante, Theoretical and Numerical Combustion, www.cerfacs.fr/elearning, 3rd edition, 2011.

[46] J. O. Hirschfelder, C. F. Curtiss, R. B. Bird, et al., Molecular theory of gases and liquids, volume 26, Wiley New York, 1954.

[47] D. Veynante, T. Schmitt, M. Boileau, V. Moureau, Analysis of dynamic models for turbulent premixed combustion, in: Proceedings of the Summer Program, Center for Turbulence Research, Stanford Univ., 2012, pp. $387-396$.

[48] F. Gouldin, K. Bray, J.-Y. Chen, Chemical closure model for fractal flamelets, Combust. Flame 77 (1989) 241-259. 
[49] Ö. L. Gülder, G. J. Smallwood, Inner cutoff scale of flame surface wrinkling in turbulent premixed flames, Combust. Flame 103 (1995) $107-114$.

[50] D. Veynante, V. Moureau, Analysis of dynamic models for large eddy simulations of turbulent premixed combustion, Combust. Flame (2015).

[51] T. Poinsot, D. Haworth, G. Bruneaux, Direct simulation and modeling of flame-wall interaction for premixed turbulent combustion, Combust. Flame 95 (1993) 118-132.

[52] G. Bruneaux, T. Poinsot, J. Ferziger, Premixed flame--wall interaction in a turbulent channel flow: budget for the flame surface density evolution equation and modelling, J. Fluid Mech. 349 (1997) 191-219.

[53] T. Schonfeld, M. Rudgyard, Steady and unsteady flow simulations using the hybrid flow solver AVBP, AIAA J. 37 (1999) 1378-1385.

[54] T. J. Poinsot, S. Lele, Boundary conditions for direct simulations of compressible viscous flows, J. Comput. Phys. 101 (1992) 104-129.

[55] O. Colin, M. Rudgyard, Development of high-order Taylor-Galerkin schemes for LES, J. Comput. Phys. 162 (2000) 338-371.

[56] F. Nicoud, F. Ducros, Subgrid-scale stress modelling based on the square of the velocity gradient tensor, Flow Turb. Combust. 62 (1999) 183-200.

[57] L. Selle, G. Lartigue, T. Poinsot, R. Koch, K.-U. Schildmacher, W. Krebs, B. Prade, P. Kaufmann, D. Veynante, Compressible large 
eddy simulation of turbulent combustion in complex geometry on unstructured meshes, Combust. Flame 137 (2004) 489-505.

[58] D. Veynante, R. Knikker, Comparison between LES results and experimental data in reacting flows, J. Turb. (2006).

[59] V. Caux-Brisebois, A. M. Steinberg, C. M. Arndt, W. Meier, Thermoacoustic velocity coupling in a swirl stabilized gas turbine model combustor, Combust. Flame 161 (2014) 3166-3180.

[60] A. Steinberg, C. Arndt, W. Meier, Parametric study of vortex structures and their dynamics in swirl-stabilized combustion, Proc. Combust. Inst. 34 (2013) 3117-3125.

[61] G. Lartigue, U. Meier, C. Bérat, Experimental and numerical investigation of self-excited combustion oscillations in a scaled gas turbine combustor, Appl. Therm. Eng. 24 (2004) 1583-1592. 


\section{List of Figures}

1 Sketch of the Preccinsta burner and the corresponding computational domain and mesh (top). Bottom: zoom on the combustion chamber. . . . . . . . . . . . . . . . . . 38

2 Instantaneous field of the filtered progress variable $\widetilde{c}=0.5$ iso-surface colored by the $\beta$ parameter (local approach, $L_{0.83}$ ). 38

3 Instantaneous snapshots of the heat release field when using global $\left(G_{0.83}\right.$, left) and local $\left(L_{0.83}\right.$, right $)$ dynamic approaches. The red color indicates the maximum heat release observed when using the local model parameter $\left(2000 \mathrm{MW} / \mathrm{m}^{3}\right)$, while blue corresponds to $1 \%$ of this maximum value. . . . . . . . . 39

4 Mean (top) and resolved RMS (bottom) $\mathrm{CH}_{4}[\%]$ transverse mass fraction profiles at five downstream locations for simulation $L_{0.83}$. Symbols: experimental data from [1] (o): Favre measurements; ( $\square$ ): Reynolds measurements; (- - - ) QuasiReynolds filtered quantity (Eqs. 14-15); (-) Favre filtered quantity (Eqs. 16-17). . . . . . . . . . . . . . . . . . . 40

5 Favre mean (top) and resolved RMS (bottom) $\mathrm{CO}_{2}[\%]$ transverse mass fraction profiles at five downstream locations. (o): Favre measurements; (-) $L_{0.83} ;$ (- - - ) $G_{0.83} ;$ (- - - ) $C_{0.83}$. . 41

6 Favre mean (top) and resolved RMS (bottom) temperature $T[K]$ transverse profiles at five downstream locations. (o): Favre measurements; (-) $L_{0.83} ;\left(\mathbf{- ~ - ~ - ~ ) ~} G_{0.83} ;\left(\mathbf{-}\right.\right.$. - ) $C_{0.83} . \quad$. 42 
7 Favre mean $C O$ [\%] transverse mass fraction profiles at five downstream locations. (o): Favre measurements; (-) $L_{0.83}$; (- - - ) $G_{0.83} ;$ (- - - ) $C_{0.83} \ldots \ldots \ldots \ldots$

8 Temporal evolution of the global parameter $\beta(t)(-\bullet-)$, local average model parameter $\bar{\beta}(t)$, Eq. $18(-\bullet-)$ and its fluctuation $\beta^{\prime}(t)$, Eq. $19(-)$. . . . . . . . . . . . . 43

$9 \quad$ Instantaneous LES resolved fields and corresponding test-filtered quantities. (left) Iso- $\widetilde{c}$ surface, (right) Iso- $\widehat{\widetilde{c}}$ surface when $\gamma=1.5 .44$

10 Normalized filtered flame surface, $\widehat{S}_{r} / S_{r}$, as a function of $\gamma$ (Eq. 6) comparing test and combustion filter widths in loglog scales. Surfaces are computed from a single instantaneous field using Eqs (20) and (21) where the volume $\mathcal{V}$ is either the entire domain, the upstream $(h<15 \mathrm{~mm})$ or the downstream ( $h>15 \mathrm{~mm}$ ) half of the flame. The $\beta$ values corresponds to the mean curve slopes. . . . . . . . . . . . . . . . . . . . . . . 44

11 Probability density function of the model parameter $\beta$ for the entire domain (top), $h<15 \mathrm{~mm}$ (bottom left) and $h>15$ $\mathrm{mm}$ (bottom right), where $h$ is the downstream location, for the quiet flame corresponding to Fig. 2 (case $L_{0.83}$ ). Data are extracted from the same timestep than Fig. 10. . . . . . . . . 45

12 Temporal evolution of the total heat release per unit volume for simulations using local $\left(L_{0.70}, \boldsymbol{-}\right)$, global $\left(G_{0.70}, \mathbf{- ~ - ~ - ~}\right)$ dynamic and non-dynamic $\left(C_{0.70},-\right.$ - - $)$ models. . . . . . . 45 
13 Temporal evolution of the plenum pressure fluctuation $P_{p}(-$ ); the chamber pressure fluctuation $P_{c}$ (- - - ) and the total heat release ( - . - ) for simulation $L_{0.70}$ using the local dynamic model. Phases ph $1-p h 8$ of the oscillation (see Fig. 14) are also indicated. . . . . . . . . . . . . . . . 46

14 Instantaneous field of $\widetilde{c}=0.5$ iso-surface colored by the $\beta$ parameter (local approach, case $L_{0.70}$ ) for eight different phases: (a) $p h 1$, (b) $p h 2$, (c) $p h 3$, (d) $p h 4$, (e) $p h 5$, (f) $p h 6$, (g) $p h 7$ and (h) ph8 for the pulsating flame (see Fig. 13). Probability density function of the fractal model parameter $\beta$ is also plotted for each phase. The blue color corresponds to $\beta=0$ and the red color to $\beta=1$. . . . . . . . . . . . . . . . . . 4

15 Favre mean (top) and resolved RMS (bottom) $\mathrm{CH}_{4}[\%]$ transverse mass fraction profiles. (o): Favre measurements [1]; (-

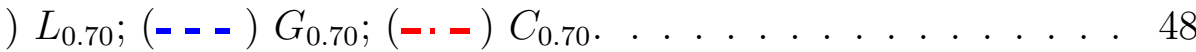

16 Favre mean (top) and resolved RMS (bottom) transverse axial velocity $[\mathrm{m} / \mathrm{s}]$ profiles. (o): Favre measurements [1]; () $L_{0.70} ;(-$ - $) G_{0.70} ;(-.-) C_{0.70} \ldots \ldots \ldots$

17 Normalized filtered surface flame as a function of the ratio of test and combustion filter sizes in log-log scales for eight different phases, $p h 1-p h 8$, corresponding to Fig. 14 (case $\left.L_{0.70}\right) \ldots \ldots \ldots \ldots \ldots$ 
18 Temporal evolution of the mean model parameter $\bar{\beta}$ (Eq. 18, -), its fluctuation $\beta^{\prime}$ (Eq. 19, - - - ), and the total heat release $\left(-{ }^{-}-\right)$for simulation using the local dynamic model $\left(\right.$ case $\left.L_{0.70}\right) \ldots \ldots \ldots \ldots \ldots$

19 Temporal evolution of the global parameter $\beta$ ( - ) and the total heat release (- - ) for simulation using the global dynamic model (case $\left.G_{0.70}\right)$. . . . . . . . . . . . . . 51

20 Temporal evolution of the total heat release per unit volume for the pulsating flame using the local dynamic model. () standard mesh; (- - ) fine mesh. . . . . . . . . . . . . 51

21 Favre mean (top) and resolved RMS (bottom) $\mathrm{CH}_{4}[\%]$ transverse mass fraction profiles at five downstream locations. (o): Favre measurements [1]; (—) standard mesh; (- - - ) fine mesh 52 

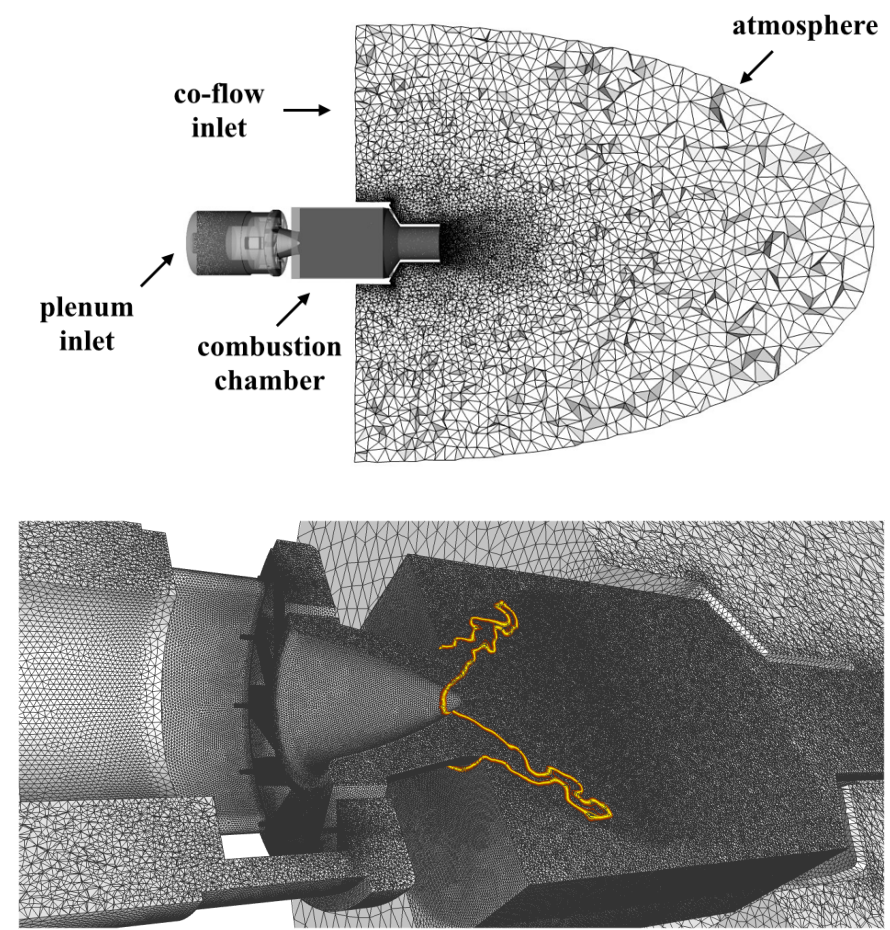

Figure 1: Sketch of the Preccinsta burner and the corresponding computational domain and mesh (top). Bottom: zoom on the combustion chamber.

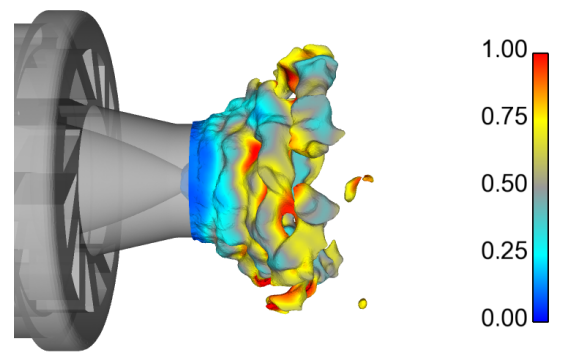

Figure 2: Instantaneous field of the filtered progress variable $\widetilde{c}=0.5$ isosurface colored by the $\beta$ parameter (local approach, $L_{0.83}$ ). 


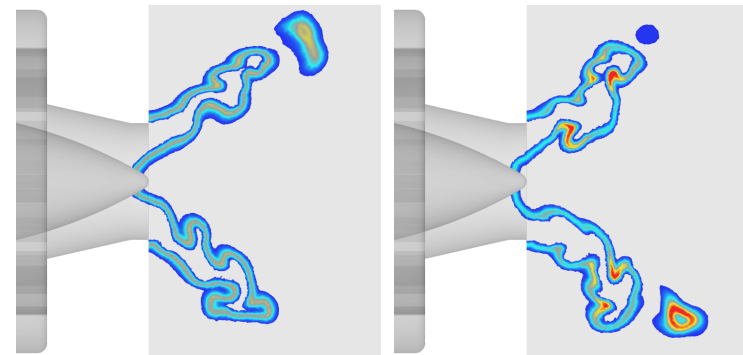

Figure 3: Instantaneous snapshots of the heat release field when using global $\left(G_{0.83}\right.$, left $)$ and local $\left(L_{0.83}\right.$, right $)$ dynamic approaches. The red color indicates the maximum heat release observed when using the local model parameter $\left(2000 \mathrm{MW} / \mathrm{m}^{3}\right)$, while blue corresponds to $1 \%$ of this maximum value. 

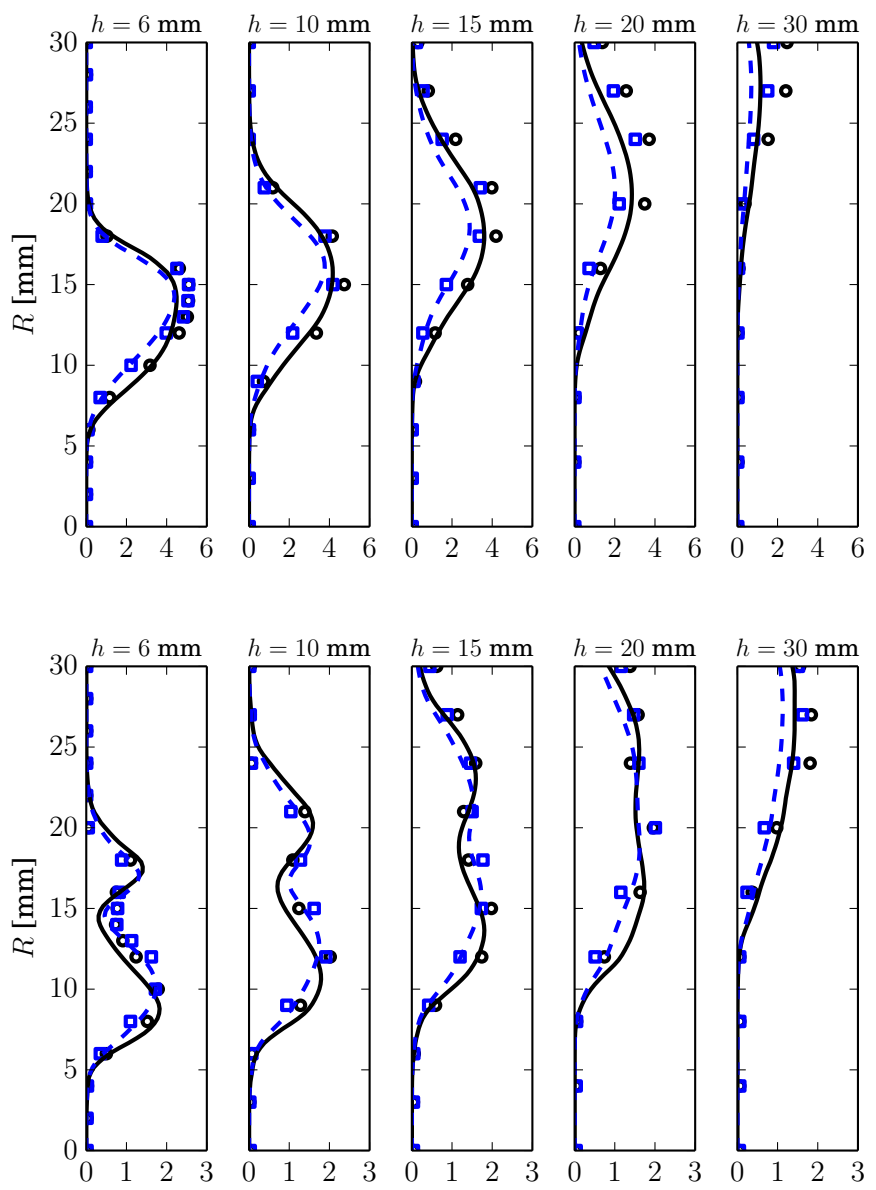

Figure 4: Mean (top) and resolved RMS (bottom) $\mathrm{CH}_{4}[\%]$ transverse mass fraction profiles at five downstream locations for simulation $L_{0.83}$. Symbols: experimental data from [1] (o): Favre measurements; ( $\square$ ): Reynolds measurements; (- - - ) Quasi-Reynolds filtered quantity (Eqs. 14-15); () Favre filtered quantity (Eqs. 16-17). 

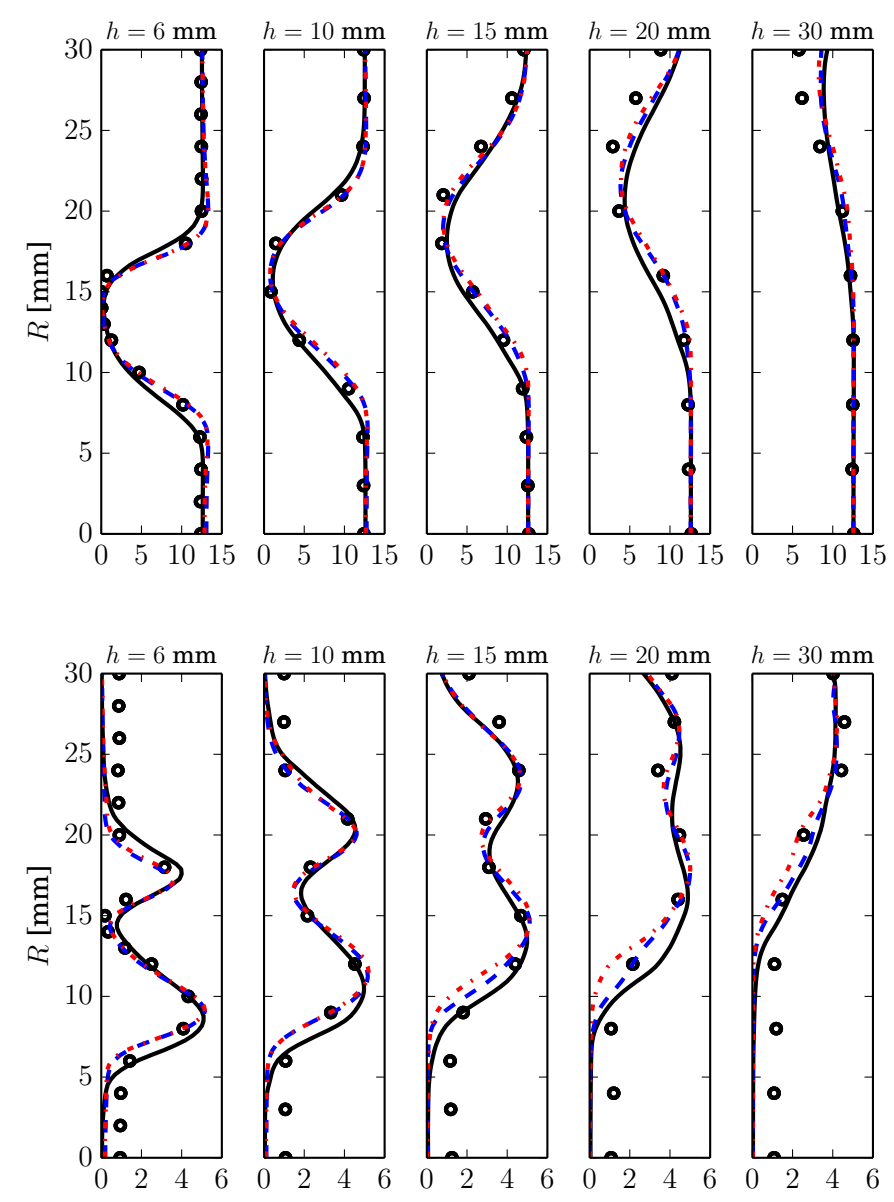

Figure 5: Favre mean (top) and resolved RMS (bottom) $\mathrm{CO}_{2}[\%]$ transverse mass fraction profiles at five downstream locations. (o): Favre measurements; (-) $L_{0.83} ;\left(-\right.$ - - ) $G_{0.83} ;\left(-\right.$ - ) $C_{0.83}$. 

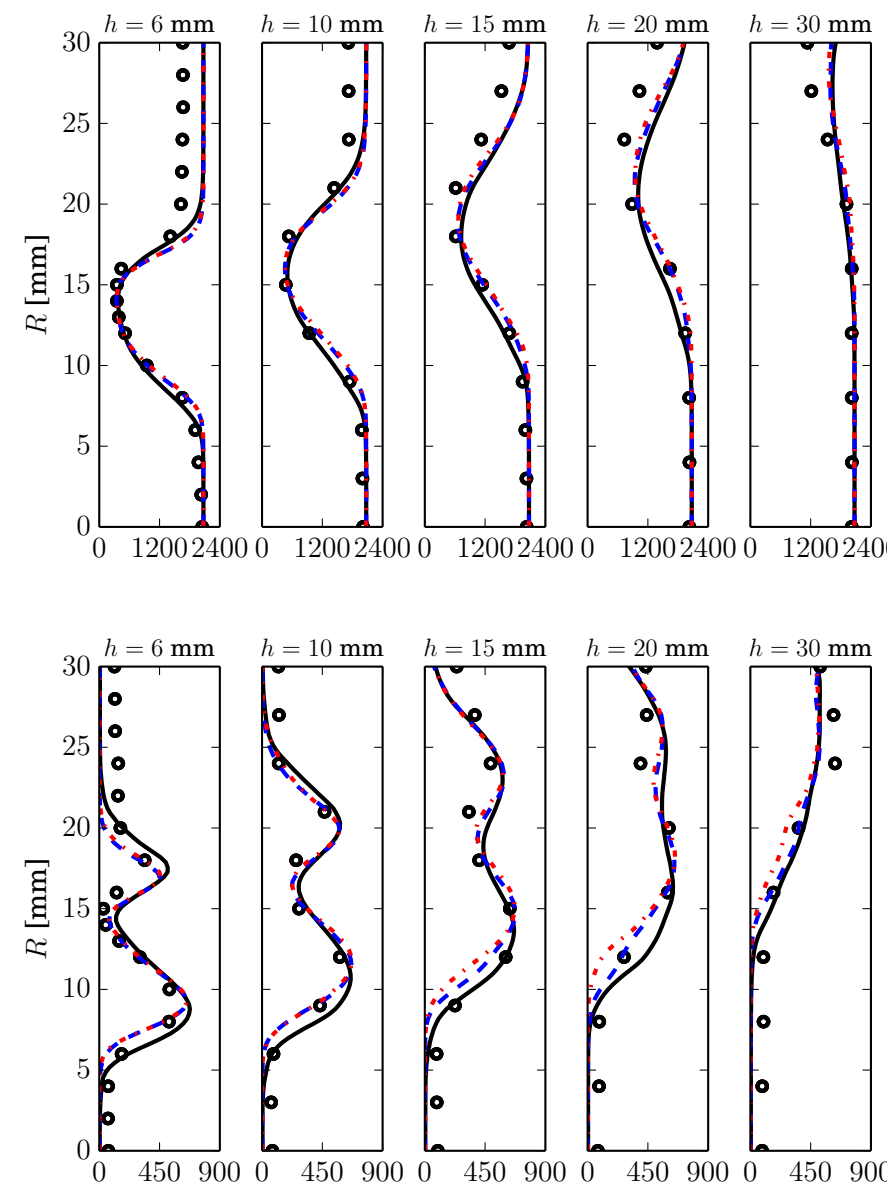

Figure 6: Favre mean (top) and resolved RMS (bottom) temperature $T[K]$ transverse profiles at five downstream locations. (o): Favre measurements; (—) $L_{0.83} ;\left(-\right.$ - - ) $G_{0.83} ;\left(-\right.$ - ) $C_{0.83}$. 


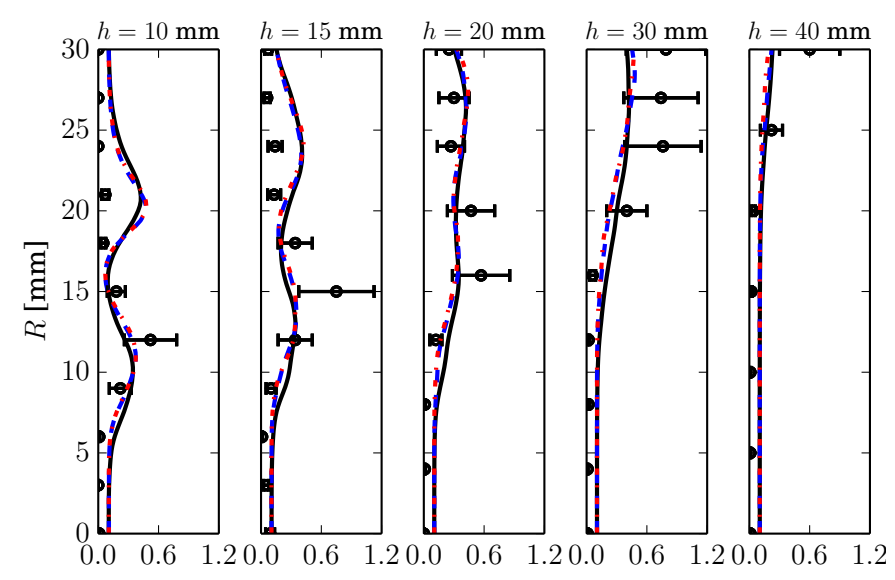

Figure 7: Favre mean $C O[\%]$ transverse mass fraction profiles at five downstream locations. (o): Favre measurements; (—) $L_{0.83} ;\left(\right.$ - - - ) $G_{0.83} ;$ (- . ) $C_{0.83}$.

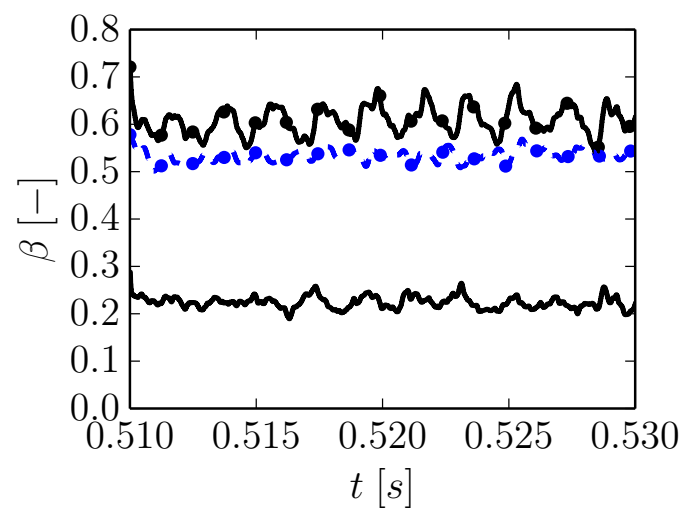

Figure 8: Temporal evolution of the global parameter $\beta(t)(-\bullet-)$, local average model parameter $\bar{\beta}(t)$, Eq. $18(-\bullet-)$ and its fluctuation $\beta^{\prime}(t)$, Eq. 19 (一). 


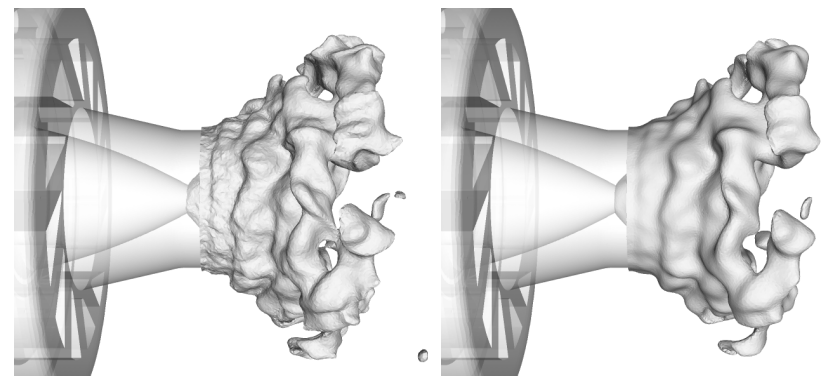

Figure 9: Instantaneous LES resolved fields and corresponding test-filtered quantities. (left) Iso- $\widetilde{c}$ surface, (right) Iso- $\widehat{\widetilde{c}}$ surface when $\gamma=1.5$.

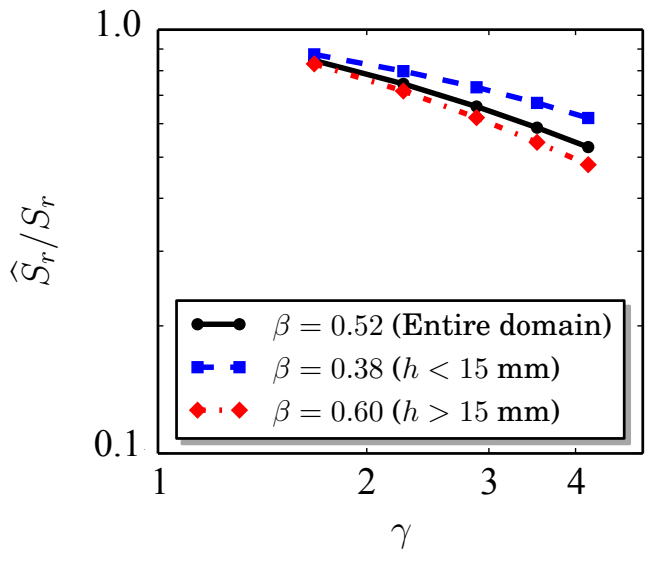

Figure 10: Normalized filtered flame surface, $\widehat{S}_{r} / S_{r}$, as a function of $\gamma$ (Eq. 6) comparing test and combustion filter widths in log-log scales. Surfaces are computed from a single instantaneous field using Eqs (20) and (21) where the volume $\mathcal{V}$ is either the entire domain, the upstream $(h<15 \mathrm{~mm})$ or the downstream $(h>15 \mathrm{~mm})$ half of the flame. The $\beta$ values corresponds to the mean curve slopes. 

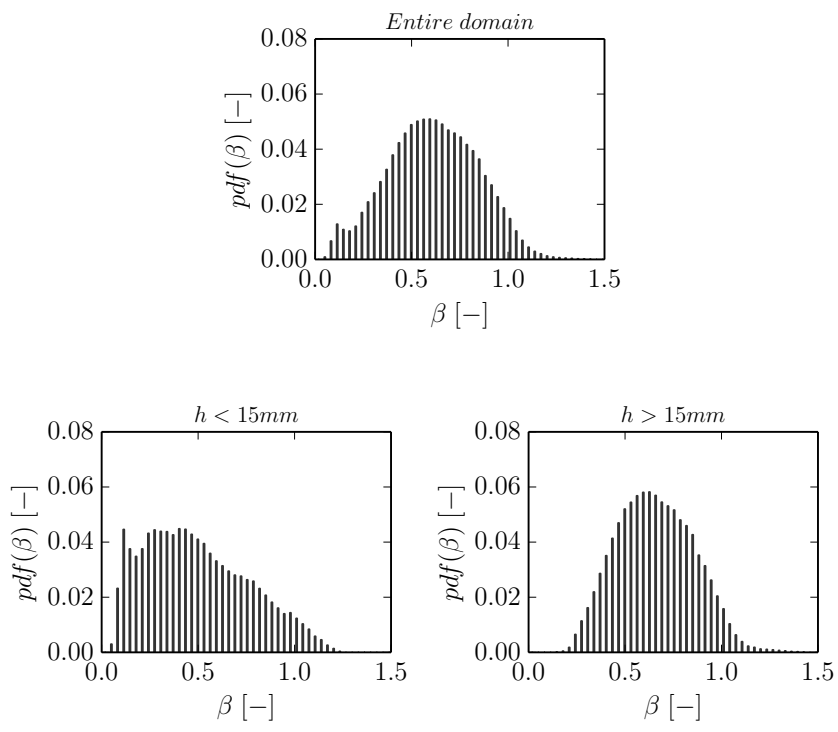

Figure 11: Probability density function of the model parameter $\beta$ for the entire domain (top), $h<15 \mathrm{~mm}$ (bottom left) and $h>15 \mathrm{~mm}$ (bottom right), where $h$ is the downstream location, for the quiet flame corresponding to Fig. 2 (case $L_{0.83}$ ). Data are extracted from the same timestep than Fig. 10.

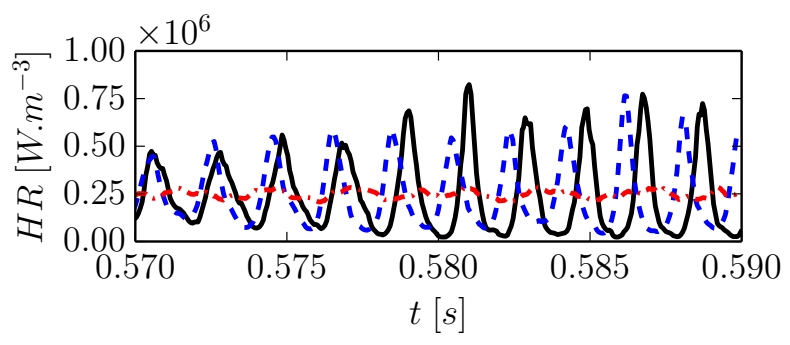

Figure 12: Temporal evolution of the total heat release per unit volume for simulations using local $\left(L_{0.70},-\right)$, global $\left(G_{0.70},-\mathbf{-}\right)$ dynamic and nondynamic $\left(C_{0.70},-\cdot-\right)$ models. 


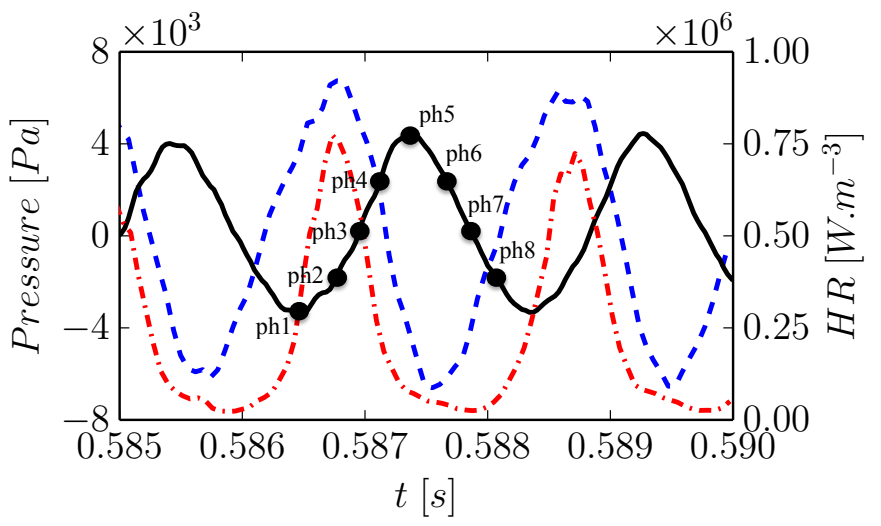

Figure 13: Temporal evolution of the plenum pressure fluctuation $P_{p}(-)$; the chamber pressure fluctuation $P_{c}(\mathbf{-}-\mathbf{-})$ and the total heat release (- : - ) for simulation $L_{0.70}$ using the local dynamic model. Phases $p h 1-p h 8$ of the oscillation (see Fig. 14) are also indicated. 
(a)

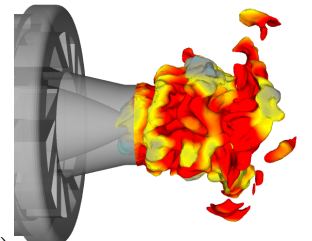

(a)

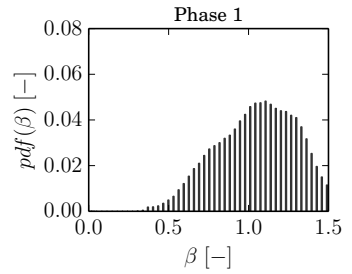

(b)
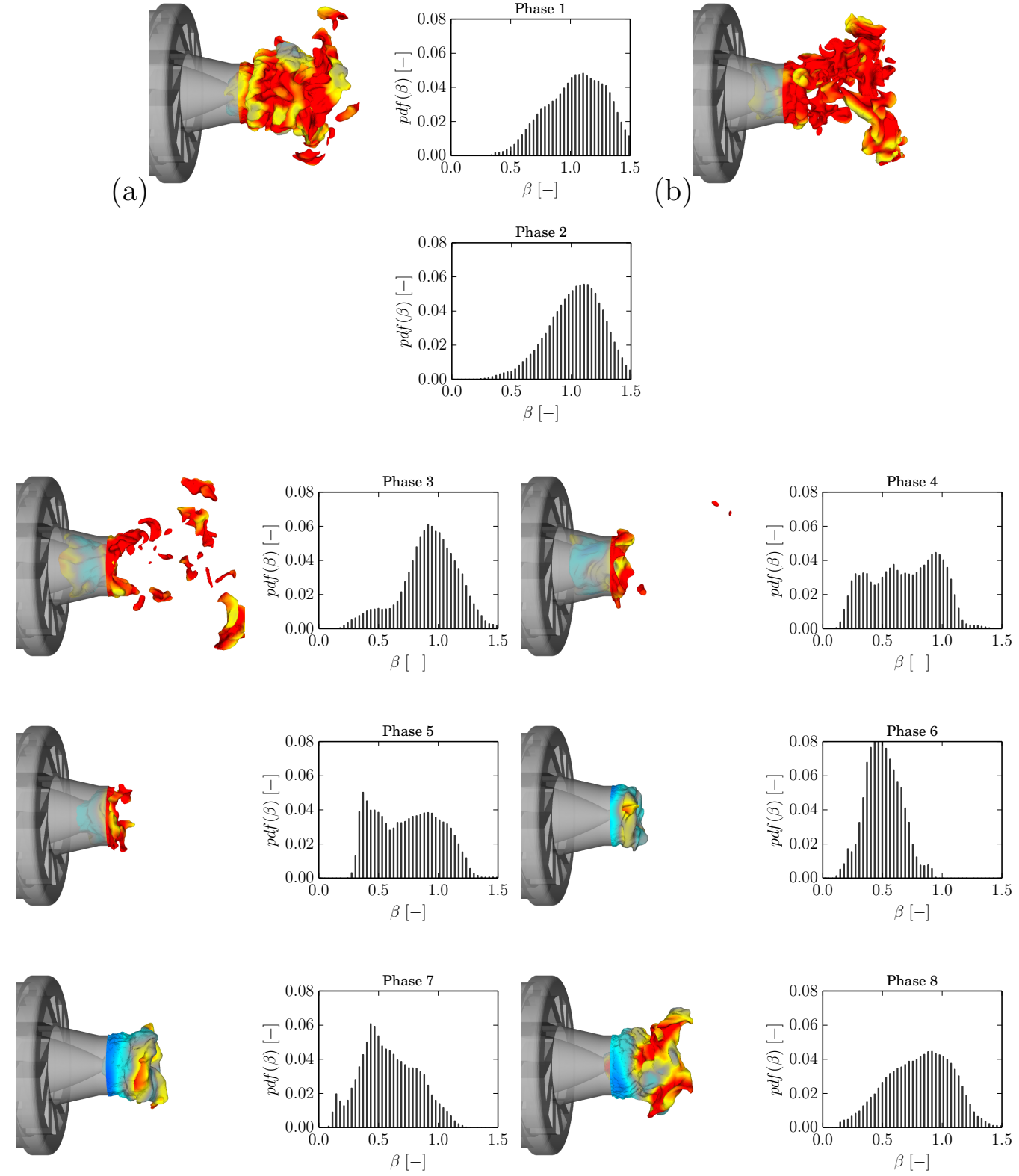

Figure 14: Instantaneous field of $\widetilde{c}=0.5$ iso-surface colored by the $\beta$ parameter (local approach, case $L_{0.70}$ ) for eight different phases: (a) ph1, (b) ph2, (c) $p h 3$, (d) $p h 4$, (e) $p h 5$, (f) $p h 6$, (g) $p h 7$ and (h) $p h 8$ for the pulsating flame (see Fig. 13). Probability density function of the fractal model parameter $\beta$ is also plotted for each phase. The bitue color corresponds to $\beta=0$ and the red color to $\beta=1$. 

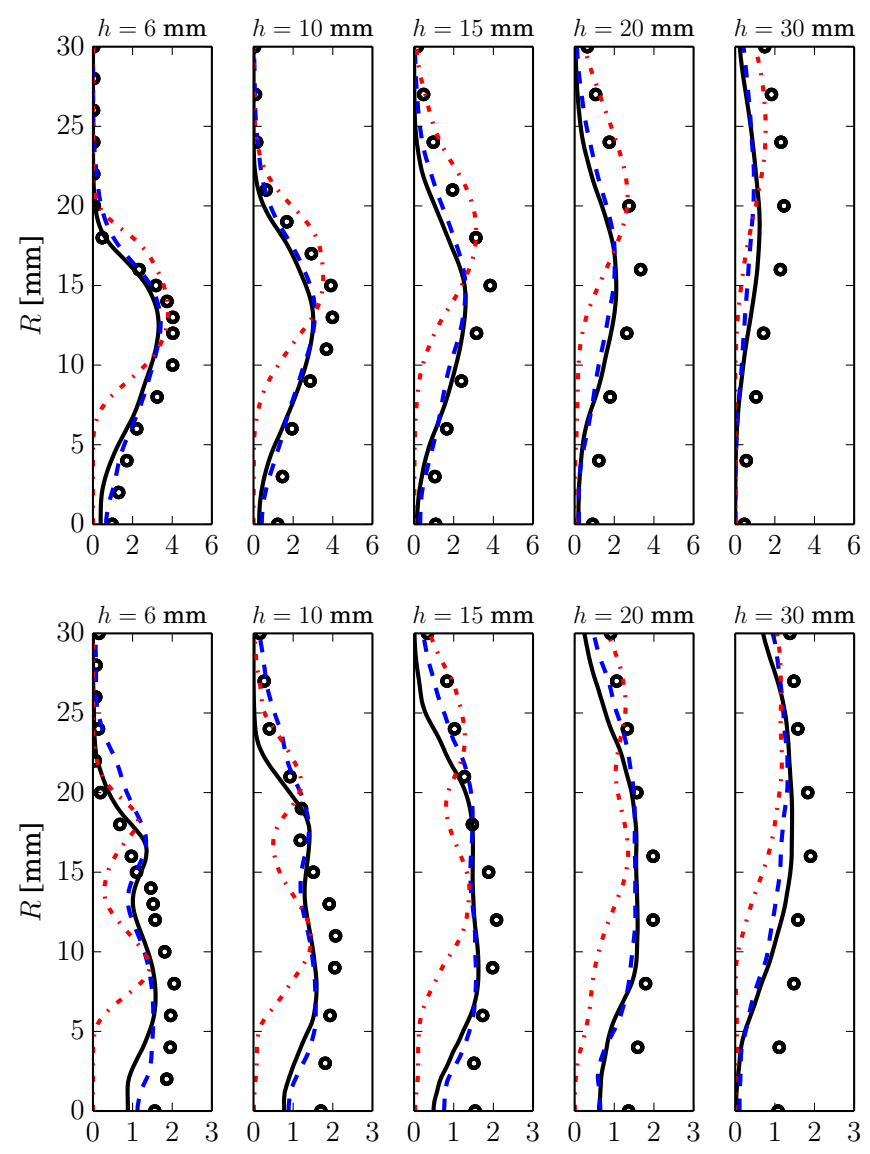

Figure 15: Favre mean (top) and resolved RMS (bottom) $\mathrm{CH}_{4}$ [\%] transverse mass fraction profiles. (o): Favre measurements [1]; (-) $L_{0.70} ;(\mathbf{-}-\mathbf{-}$ ) $G_{0.70} ;\left(-\cdot-\right.$ ) $C_{0.70}$. 

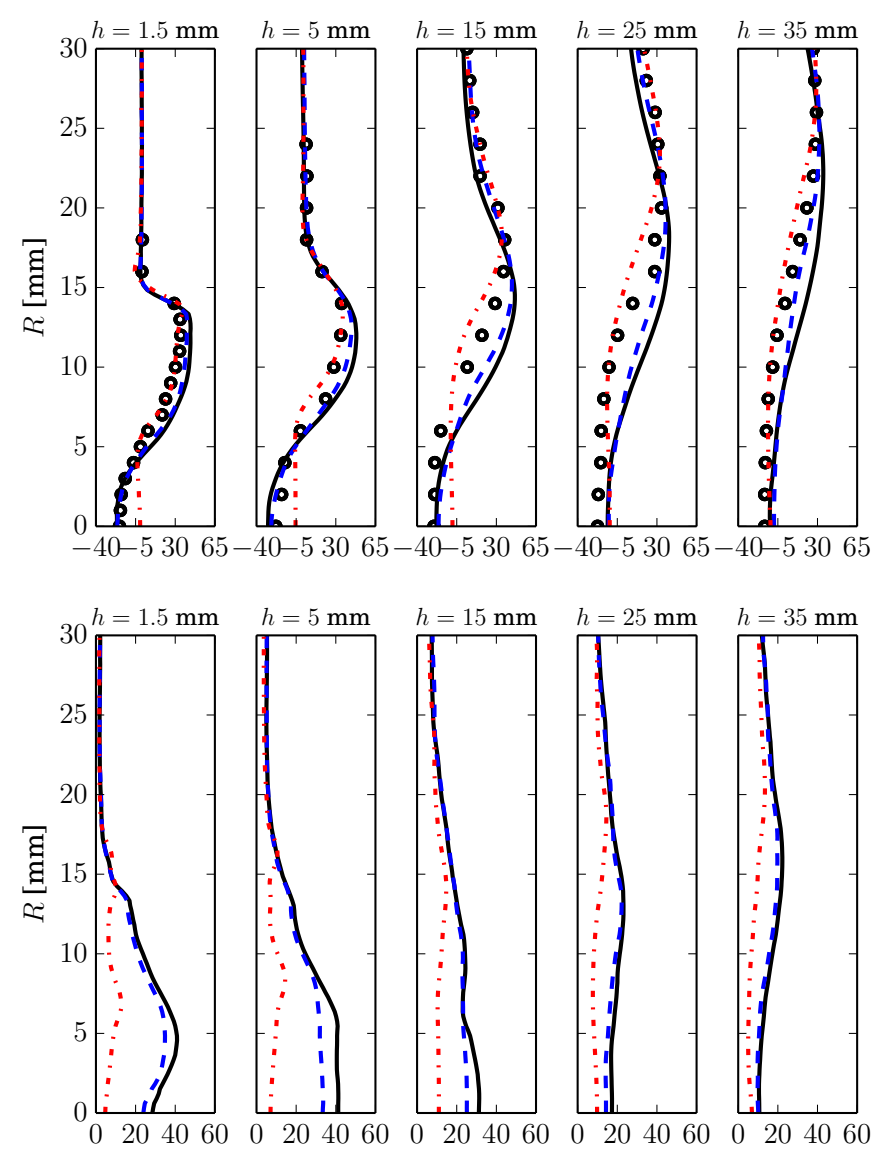

Figure 16: Favre mean (top) and resolved RMS (bottom) transverse axial velocity $[\mathrm{m} / \mathrm{s}]$ profiles. (o): Favre measurements $[1] ;(-) L_{0.70} ;\left(\mathbf{- ~ - ~ - ~ ) ~} G_{0.70}\right.$; (- - - ) $C_{0.70}$. 


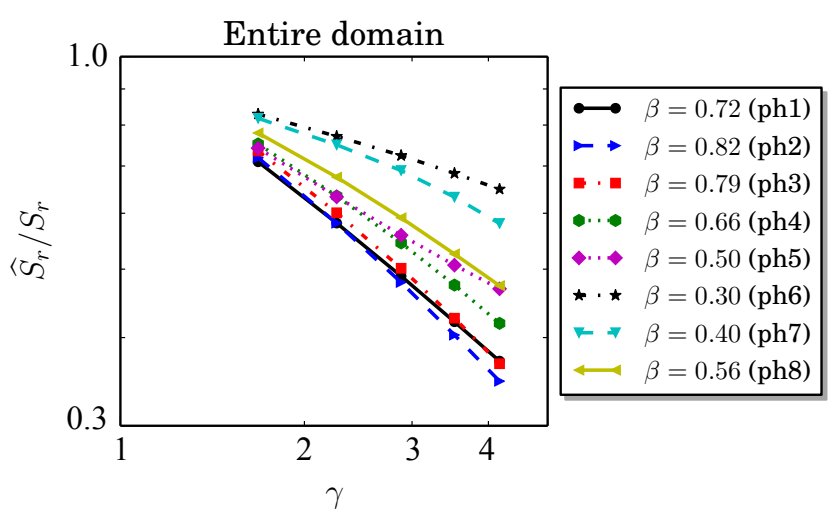

Figure 17: Normalized filtered surface flame as a function of the ratio of test and combustion filter sizes in log-log scales for eight different phases, $p h 1-p h 8$, corresponding to Fig. 14 (case $L_{0.70}$ ).

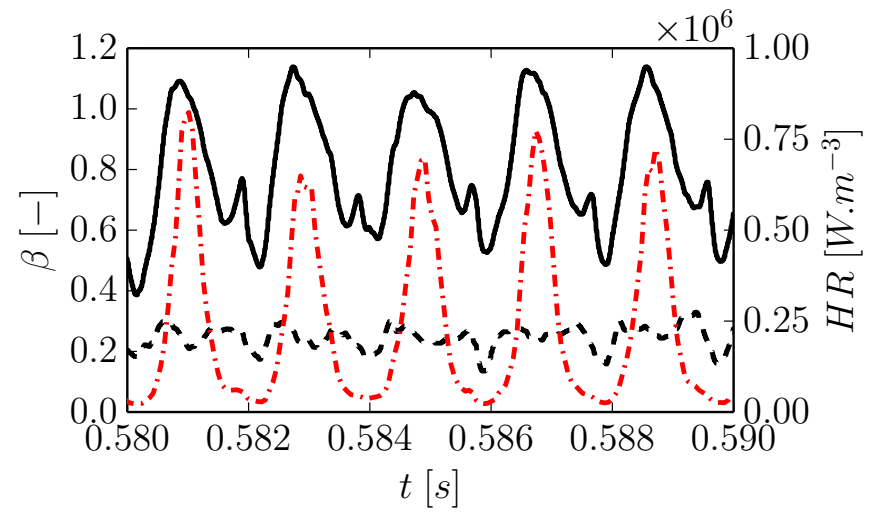

Figure 18: Temporal evolution of the mean model parameter $\bar{\beta}$ (Eq. 18, ), its fluctuation $\beta^{\prime}$ (Eq. 19, - - - ), and the total heat release (- - - ) for simulation using the local dynamic model (case $L_{0.70}$ ). 


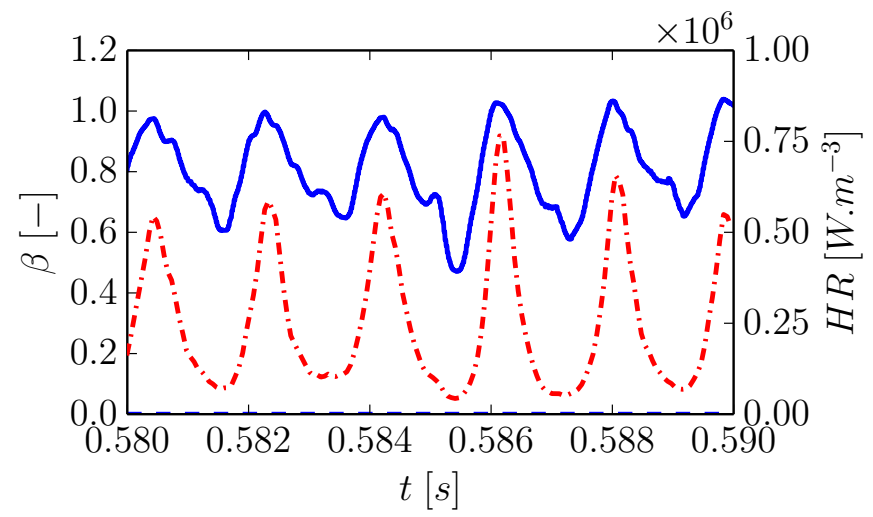

Figure 19: Temporal evolution of the global parameter $\beta(-)$ and the total heat release (- : - ) for simulation using the global dynamic model (case $\left.G_{0.70}\right)$

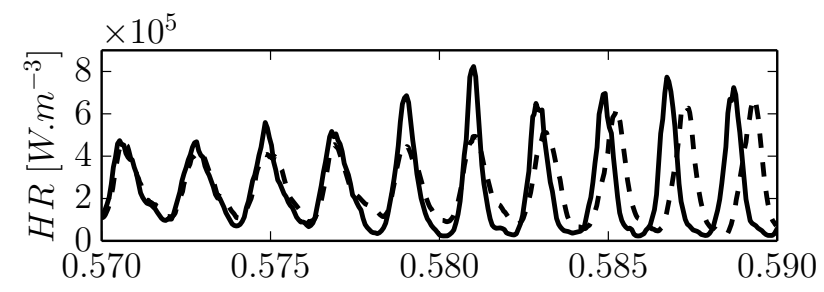

Figure 20: Temporal evolution of the total heat release per unit volume for the pulsating flame using the local dynamic model. (_- ) standard mesh; (- - ) fine mesh. 

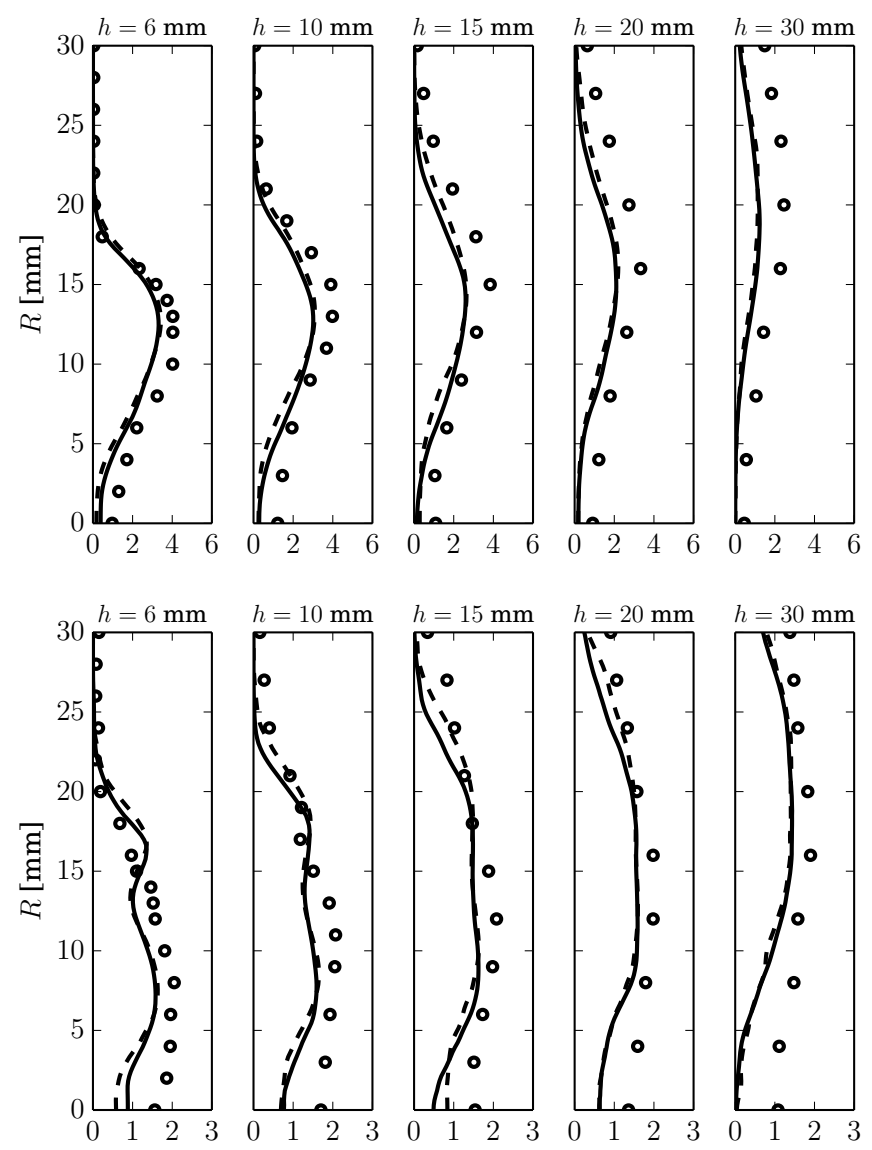

Figure 21: Favre mean (top) and resolved RMS (bottom) $\mathrm{CH}_{4}$ [\%] transverse mass fraction profiles at five downstream locations. (o): Favre measurements [1]; (-) standard mesh; (- - - ) fine mesh 